\title{
Foundations of Applied Mathematics I
}

\author{
Jeffrey Ketland \\ University of Warsaw \\ jeffreyketland@gmail.com \\ Forthcoming in Synthese
}

November 23, 2020

\begin{abstract}
This paper aims to study the foundations of applied mathematics, using a for-

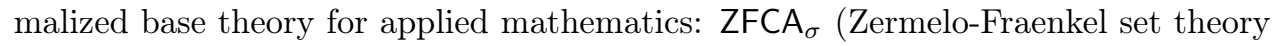
(with Choice) with atoms, where the subscript used refers to a signature specific to the application. Examples are given, illustrating the following five features of applied mathematics: comprehension principles, application conditionals, representation hypotheses, transfer principles and abstract equivalents.
\end{abstract}

\section{Contents}

1 Mathematicized Theories 2

2 How The Laws of Set Theory Apply 8

3 A Base Theory for Applied Mathematics 9

4 Comprehension and Set Abstracts $\quad 17$

5 Abstract Counterparts: Equivalence Modulo Mathematics 20

$\begin{array}{lll}6 & \text { Systems and Equivalences } & 21\end{array}$

$\begin{array}{lll}7 & \text { Application Conditionals } & \mathbf{3 0}\end{array}$

8 Representation and Transfer $\quad 34$

$\begin{array}{llr}9 & \text { Summary } & 37\end{array}$

$\begin{array}{ll}\text { A Four-Sorted System } & 38\end{array}$ 


\section{Mathematicized Theories}

Standard scientific theory supplies much of the information it supplies about physical entities only indirectly, by way of apparatus pertaining to supposed relationships of physical entities to supposed mathematical entities and supposed classifications of and relationships among the supposed mathematical entities themselves. As much of what science says about observable entities is "theory-laden", so much of what science says about concrete entities (observable or theoretical) is "abstraction-laden". (Burgess \& Rosen (1997): 84)

\subsection{The Logical Structure of Applied Mathematics}

This paper aims to study the foundations of applied mathematics. Perhaps contrary to current fashions, I treat applied mathematics as taking place within a certain formalized system: specifically, Zermelo-Fraenkel set theory (with Choice) with atoms, over an application signature $\sigma$. This formalized system is denoted ZFCA $_{\sigma}$. We shall think of it as a standard base theory for applied mathematics.

The subscript " $\sigma$ " on "ZFCA $\sigma$ " denotes what I shall call the application signature. This signature may contain predicates and function symbols which relate only "atoms"/"urelements" and also may contain predicates which relate atoms to sets and, more generally, to other abstract/mathematical objects. Since $\sigma$ can be varied, there is a base theory ZFCA $\sigma$ for each signature $\sigma$, rather than a single overall base theory.

There is no great originality in aiming to identify a base theory for applied mathematics and focusing on something like ZFCA $\sigma$ as exemplar. There are related variations: higherorder logic \& type theory. Once we have some sort of framework set up, we can aim to implement insights from many mathematicians, logicians, scientists and philosophers over the years in connection with the logical analysis of applied mathematics. ${ }^{1}$ In particular, we wish to carefully examine how mixed predicates are integrated within scientific and mathematical reasoning - for, without mixed predicates, there is no applied mathematics.

When this issue is properly understood, we begin to obtain a good account of the central features that make applied mathematics tick. I single out these five important features:

\begin{tabular}{ll}
\hline Comprehension principles & $\exists X \forall x(x \in X \leftrightarrow \phi(x))$. \\
Application conditionals & $\phi_{1}(X, \ldots) \rightarrow \phi_{2}(X, \ldots)$. \\
Representation hypotheses & $F: P \cong M$. \\
Transfer principles & $\phi(P) \leftrightarrow \phi(M)$. \\
Abstract equivalents & $\phi \leftrightarrow \phi^{\text {abs }}$.
\end{tabular}

1 Examples include: Frege (1884), Hilbert (1899), Hölder (1901), Russell (1903), Einstein (1921), Carnap (1928), Ramsey (1929), McKinsey et al. (1953), Tarski (1959), Suppes (1960), Putnam (1967), Putnam (1971), Krantz et al. (1971), Sneed (1971), Stegmüller (1976), Field (1980), Burgess (1984), Lewis (1991), Burgess \& Rosen (1997), Shapiro (1997), Resnik (1997), Steiner (1998), Ketland (1998), Colyvan (2001), Leng (2010), Ketland (2011), Bueno \& Colyvan (2011), Andréka et al. (2012), Pincock (2012), Mycielski (2013), Halvorson (2012), Halvorson (2016), Bueno \& French (2018). 
These features, and how they appear in mathematicized theories and applications, are explained in more detail in the sections below. ${ }^{2}$

\subsection{Mixed Predicates; Mixed \& Impure Objects}

The standard number systems are $\mathbb{N}, \mathbb{Z}, \mathbb{Q}, \mathbb{R}$ and $\mathbb{C}$. The elements of these are sui generis "pure" mathematical objects, in some intuitive sense, not easy to pin down-roughly, they don't have non-mathematical elements and don't "depend" on non-mathematical objects at all. On the other hand, when one considers sets, relations, functions and structures in general, the situation is different. This is because there may be "urelements"/"atoms". So, one may consider sets of urelements, relations amongst ur-elements and functions from urelements to, say, real numbers, and structures whose domain contains urelements. Such examples are called "impure" sets, relations, functions and structures. These are the fundamental objects of applied mathematics.

The notion of "pure set" is defined as follows (and may be generalized to relations, functions and structures). A set $A$ is called transitive just if, for any $x, y$, if $x \in y$ and $y \in A$, then $x \in A$. The transitive closure of a set $A$ is the smallest transitive set $B$ such that $A \subseteq B$. A set is pure iff its transitive closure contains no urelements/atoms (Potter (2004): 50). So an impure set (or relation) is a set whose transitive closure contains atoms. All elements of the cumulative hierarchy $V$ - the system of sets built up from $\varnothing$ by iteratively applying the power set operation, $\mathcal{P}^{\alpha}(\varnothing)$ (e.g., $\varnothing, V_{\omega}$, etc.) - are pure. But, if there are atoms/urelements, there are also sets of them too. And these generate impure sets, relations and structures. This is particularly clear in applied mathematics.

So, in addition to pure mathematical objects, one notices that the mathematical objects referred to in applied mathematics include those in the following classes:

(i) Impure MATHEMATiCAL OBJECTS: sets of "ur-elements"/"atoms", relations amongst them, and structures built up from such impure sets and relations.

(ii) Mixed mathematical objects: functions from (and relations between) "ur-elements"/"atoms" to mathematical values (e.g., elements of $\mathbb{N}, \mathbb{R}, \mathbb{C}$, etc. of vector spaces, etc.).

2 For reasons of space, I have set aside discussion of a number of closely-related topics, which cannot be dealt with adequately here. These topics are: indispensability arguments (e.g., Colyvan (2001), Melia (2000), Leng (2010), Colyvan (2019)); mathematical explanation in science (e.g., Baker (2005), Mancosu (2018)); representation/modelling (e.g., Pincock (2012), Frigg (2012)); measurement theory (e.g., Krantz et al. (1971), Roberts (1985), Narens (1985), Suppes (2002), Tal (2015)); approximation and idealization (e.g., Batterman (2002), Pincock (2012)) and recent so-called "mapping accounts" of applied mathematics (e.g., Pincock (2012), Bueno \& Colyvan (2011) and Bueno \& French (2018)). That said, some of these issues are, albeit briefly, touched on at points below. Representation and modelling are illustrated in Example 2, Section 6, Subsection 6.4, Subsection 6.5, Section 8, Example 5 and Example 6. I comment sceptically on a difficulty for the "mapping accounts" in Subsection 7.3 connected to differential equations. An example of mathematical explanation (of quantized atomic orbitals) is mentioned in Subsection 7.4. 
W. V. Quine discusses this aspect of applied mathematics in his 1986 "Reply to Charles Parsons":

Another example of applied mathematics is the use of number in measurement. In terms of physical testing procedures we describe a Fahrenheit temperature function whose arguments are place-times and whose values are real numbers. Fahrenheit temperature is a class of pairs of pure real numbers and concrete place times. Similarly distance in metres is a class of triples, each comprising one pure real number and two concrete localities.

Mathematical objects and concrete objects are thus in perpetual interplay, participating in the same triples and pairs. Mathematical vocabulary and empirical vocabulary are in perpetual interplay, participating in the same sentences. We see this already at the most primitive level of applied mathematics, when we say that there are fifty people in this room: the pure abstract number, fifty, is how many concrete people there are in this concrete room. I see pure mathematics as an integral part of our system of the world. (Quine (1986b): 398)

Example 1. Mixed functions: The magnetic field

To continue with the sort of example Quine has in mind, consider a physical field like the magnetic field $\mathbf{B}$. This is a mixed function, a map from spacetime to $\mathbb{R}^{3}$ :

$$
\mathbf{B}: \text { Spacetime } \rightarrow \mathbb{R}^{3}
$$

satisfying Maxwell's laws $(\nabla \cdot \mathbf{B}=0$, and so on). The field $\mathbf{B}$ is, to stress, a physical field. Indeed, we can measure $i t^{3}$ If a small charged particle $p$ with mass $m$ and charge $q$ is moving in the vicinity of a region with non-vanishing $\mathbf{B}$, then $p$ has a measurable physical trajectory, say $\mathbf{r}(t)$. This is determined by $\mathbf{B}$, and given, to first approximation, via the Lorentz force law (assuming the electric field is 0 ):

$$
m \frac{d^{2} \mathbf{r}}{d t^{2}}=q\left(\frac{d \mathbf{r}}{d t} \times \mathbf{B}\right) .
$$

Along with the magnetic field is its co-ordinate representation $\mathbf{B}^{\varphi}$, with respect to any chart $\varphi$ : i.e., $\mathbf{B}^{\varphi}:=\mathbf{B} \circ(\varphi)^{-1}$. While $\mathbf{B}$ is a physical field, $\mathbf{B}^{\varphi}$ is a map $\mathbb{R}^{4} \rightarrow \mathbb{R}^{3}$. Hence, a pure mathematical object. One should be careful not to conflate the mixed quantity, the physical field $\mathbf{B}$, and its pure, co-ordinate representation $\mathbf{B}^{\varphi}$.

Example 2. Impure mathematical objects

Consider the "Seven Bridges of Königsberg" problem:"

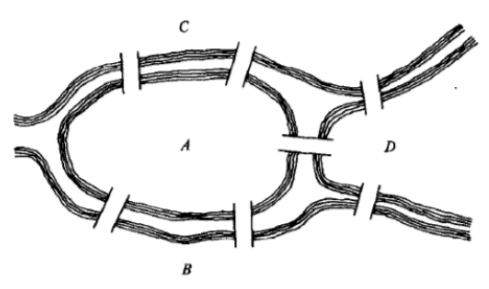

3 There are other measurement devices, like compasses, of course, Hall probes, ...

4 I took this diagram from Harary (1969) (p. 2). I return to this problem several times below. 
The diagram shows part of the city of Königsberg and the route of the Pregel River. The river's route separates the city into two large islands and two mainland areas, connected by seven bridges. The relevant locations are here called " $A$ ", " $B$ ", " $C$ " and " $D$ ". The problem was to devise a way of walking from one location, visiting each of the other three locations, covering each bridge exactly once, back to the original location.

Euler proved that there is no such route (Euler (1736)). In doing so, Euler invented graph theory and provided the first theorem proper to that area of mathematics. The relevant concept is that of a "Euler cycle" in a multigraph. ${ }^{5}$ A path in a graph is a sequence of vertices connected by edges. A cycle is a path through the graph beginning and ending at the same vertex, visiting each vertex, and going through each edge exactly once. The number of edges incident on a vertex is called its degree.

Euler discovered that a cycle is possible only if the graph is connected and the degree of each vertex is even. Roughly, the reasoning is this. Let the cycle through the vertices and edges visited be depicted as below (allowing repetitions of vertices, but no repetitions of edges):

$$
v_{1}-e_{1}-v_{2}-e_{2}-v_{3}-e_{3}-v_{4}-e_{4}-\cdots-e_{n}-v_{n}\left(=v_{1}\right)
$$

This cycle is a path containing all the vertices of the graph, and all the edges (without repetition), and final vertex is the same as the first. Examining each occurrence of a vertex, $v$, we see that there must be an incoming edge, say $e_{i n}$ and an outgoing edge, say $e_{\text {out }}$, making two edges. If the vertex $v$ occurs $n$ times in the cycle, there must be $2 n$ such distinct edges incident on $v$ in the cycle. Because each vertex in the graph must occur at least once and the graph is connected, this number is at least 2. Since these edges must be distinct and the cycle includes all edges, those $2 n$ edges must be the degree of the vertex $v$. Hence every vertex must have even degree. In fact, this condition is not merely necessary but sufficient: ${ }^{6}$

Theorem 1. A multigraph has a Euler cycle iff it is connected and each vertex has even degree.

In order to see more exactly how mathematics got applied to this problem, we need to understand how one "abstracts" - from the given data concerning the problem - a structured system. To see how this is done, let us use "land-area" to refer to the four separated regions of the city, and make the following definitions of impure mathematical objects:

5 A (simple, undirected) graph $G$ is a pair $(V, E)$ where $V$ is a set, the vertices of $G$, and $E$ is a set of distinct pairs $\{x, y\}$ drawn from $V$, the set of edges of $G$. An (undirected) multigraph is a kind of graph in which a pair of vertices may be connected by more than one edge. It may be represented as a triple $(V, E, f)$, where $V$ is the set of vertices, $E$ is a (disjoint from $V$ ) set of edges, and $f$ is a connection function, which assigns to each edge in $E$ a pair $\{x, y\}$ drawn from $V$.

6 See Harary (1969): 64. 


$$
\begin{gathered}
L:=\{x \mid x \text { is a land-area at Königsberg }\} . \\
B:=\{x \mid x \text { is a bridge at Königsberg }\} . \\
C:=\{(x, y, z) \mid x \in B \text { and } y, z \in L \text { and } x \text { connects } y \text { and } z\} .\} \\
F:=\{((x,\{y, z\}) \mid(x, y, z) \in C .\} \\
P_{K}:=(L, B, F) .
\end{gathered}
$$

Here, $P_{K}$ is the resulting structured system: it is the physical system we wish to study. ${ }^{7} P_{K}$ is not, itself, a concrete object. Rather, $P_{K}$ is itself an impure mathematical object. It is incorrect to say that, in our applied mathematics reasoning, $P_{K}$ must be "modelled" by some pure mathematical structure. ${ }^{8}$ We can simply reason about $P_{K}$ as a mathematical object.

With this in hand, we can go on to show for example (in applicable mathematics itself) that, given the specific facts about the bridges, $P_{K}$ is a connected multigraph with 4 vertices and 7 edges. Moreover, given the specific facts about the bridges, each vertex of $P_{K}$ has odd degree. And therefore, given the specific facts about the bridges and Theorem 1, it follows that there is no Euler cycle in $P_{K}$. Notice that Theorem 1 does not specify that the multigraph in question be a pure mathematical object. It applies equally well to impure mathematical objects: e.g., a structure built up from atoms/urelements. Since this example illustrates quite a few important features of applied mathematics, but without introducing orthogonal complexities related to idealization/approximation and infinity, I shall return to it a couple of times below (Section 6.4 and Example 5).

\subsection{Quantity Primitives}

Next let's write down some examples of "mixed quantity functions": 9

$$
\begin{array}{ll}
l=\ell_{\mathrm{m}}(c) & \text { the real } l \in \mathbb{R} \text { is the length in metres of the object } c \\
z=\Psi(p) & \text { the complex number } z \in \mathbb{C} \text { is the value of the wave-function } \Psi \text { at } \\
& \text { spacetime point } p
\end{array}
$$

In a formalized setting, these quantity primitives are then expressed, usually, by function symbols. In some cases, by predicates. An example of the latter might for example be: ${ }^{10}$

7 If I understand their meaning right, $P_{K}$ is the "assumed structure" that Bueno \& Colyvan (2011) refer to and the "empirical set-up" that Bueno \& French (2018) refer to.

8 Though it could be: $P_{K}$ is isomorphic to a certain abstract graph, $G_{K}$ : see Section 6.4 below.

9 The approach here is fairly close to that described in Carnap's works: Carnap (1956), Carnap (1958), Carnap (1966). See also, for example, Suppes (1960), Sneed (1971), Stegmüller (1976), Field (1980), Burgess (1984), Andréka et al. (2012).

10 This appears in the axiomatizations of relativity given by Andréka et al, who call it the "worldview relation". See especially: Andréka et al. (2006) and Andréka et al. (2012). 
$\mathrm{W}\left(\varphi, b, x_{1}, \ldots, x_{d}\right) \quad$ observer $\varphi$ coordinatizes body $b$ by coordinates $x_{1}, \ldots, x_{d}$ (in his coordinate system).

\subsection{A Classification}

Example 1 gave the example of a mixed function, the physical field B. Example 2 showed how impure sets, relations and structures turn up in applied mathematics. In the previous subsection, we gave some further examples of mixed quantity primitives.

General consideration of the kinds of mathematical objects appearing in applied mathematics suggests the following seven-fold classification of basic impure \& mixed mathematical objects: ${ }^{11}$

\begin{tabular}{ll} 
IMPURE MATHEMATICAL OBJECTS & MiXED MATHEMATICAL OBJECTS \\
\hline 1. Sets (of atoms) & 4. Mixed relations \\
2. Relations (of atoms) & 5. Charts (co-ordinate systems) \\
3. Structures (on atoms) & 6. Scales (mass, length, duration, etc.) \\
& 7. Fields (scalar, spinor, tensor, etc.)
\end{tabular}

Applied mathematics is then the business of asserting the existence of, and establishing properties of, certain impure sets, relations and structures, and certain mixed relations and functions. To be more exact, applied mathematics allows for the possibility that the sets, relations and structures referred to are impure, and the relations and functions referred to are mixed. Of course, we frequently don't know whether some set (or relation or structure) $X$ is impure, or whether some function $F$ is mixed. Indeed, free variables within the overall theory can leave this unspecified.

The beauty of applied mathematics is that it doesn't matter. For example, mathematical principles such as:

$$
\begin{gathered}
\qquad|X|+|Y|=|X \cup Y|-|X \cap Y| \\
\text { If } A \stackrel{F}{\cong} B \text {, any structural property of } A \text { is a property of } B \\
\text { If }\left(F: X \rightarrow \mathbb{C} \text { and } \ldots \text { and }(\forall x: X)\left(-a \nabla^{2}-\frac{b}{|x|}\right) \Psi(x)=E \Psi(x) \text { and } E<0 \text {, then } E=-\frac{c}{n^{2}} \text {, with } n \in \mathbb{N}_{>0}\right.
\end{gathered}
$$

hold irrespective of whether the sets $X, Y$ are pure/impure, and irrespective of whether the spaces $A, B$ are built from atoms or not, and irrespective of whether the set (or structure) $X$ is impure and the function $F: X \rightarrow \mathbb{C}$ is mixed or not. ${ }^{12}$ To apply $\forall X \forall Y(|X|+|Y|=|X \cup Y|-|X \cap Y|)$, we use comprehension on ur-element predicates to form set terms, like " $\{x \mid \operatorname{cat}(x)\}$ " and " $\{x \mid \operatorname{dog}(x)\}$ ", and instantiate the set quantifiers on these terms to obtain:

$$
|\{x \mid \operatorname{cat}(x)\}|+|\{x \mid \operatorname{dog}(x)\}|=|\{x \mid \operatorname{cat}(x)\} \cup\{x \mid \operatorname{dog}(x)\}|-|\{x \mid \operatorname{cat}(x)\} \cap\{x \mid \operatorname{dog}(x)\}|
$$

\footnotetext{
11 The point of the qualification "basic" is that further impure and mixed objects can then be defined by standard mathematical constructions: pairs, products, etc.

12 The first principle is routinely used in applications of arithmetic. See Section 8 for more on the second principle. I return to the third theorem in Section 7: Example 4 and Subsections 7.3 and 7.4.
} 


\section{How The Laws of Set Theory Apply}

Set theory is applied to non-mathematical matters via examples such as those below:

(1) The set of elephants is a subset of the set of mammals.

(2) The cardinal of the set of left-handed people is less than the cardinal of the set of right-handed people.

(3) There is no injective function from the set of people in this room to the set of handouts that I have printed.

(4) Napoleon is not an element of the set of all humans which contains Quine and is closed under the relation "parent-of".

However, many standard presentations of axiomatic set theory tend to ignore applications to non-mathematical subject matter. The reason is that more or less all core mathematics may be developed within ZFC without any assumption as to the existence of "atoms" or "urelements" or "individuals". So contemporary discussions incorporating atoms or urelements are less common. Within set theory itself, set theories with atoms derive their interest for reasons connected to independence proofs. In that connection, a closely related system is the one that Thomas Jech discusses (lacking Choice), ZFA, in his classic set theory textbook:

In order to describe this method, we introduce the theory ZFA, set theory with atoms. In addition to sets, ZFA has additional objects called atoms. These atoms do not have any elements themselves but can be collected into sets. ... The effect of atoms is that the universe is no longer obtained by iterated power set operation from the empty set. In ZFA, the universe is built up from atoms. (Jech (2002): 250)

That said, there does exist a literature which includes formulations of set theory with urelements/atoms. Historically, this was the standard approach-including both the axiomatic system given by Ernst Zermelo in 1908 and the type theories given by Bertrand Russell from 1903 on and the Church-style type theories given by Alonzo Church from 1940 on. $^{13}$

Moreover, from the point of view of applicability, the major difficulty with a pure set theory is that, since it doesn't permit sets of atoms/urelements/individuals, it's inapplicable. Commenting briefly on this in his review of Potter (2004), Timothy Bays writes:

\footnotetext{
13 Zermelo (1908), Russell (1903), Russell (1908), Russell \& Whitehead (1912). Quine (1956) discusses Zermelo's set theory with atoms (individuals, as Quine calls them: Quine suggests individuals be defined as being "self-membered"). Church's type theories were formulated with individuals at the base (Church (1940)). More recent examples include Quine's set theory with urelements, NFU (e.g., Jensen (1968), Forster (1995), Holmes (1998)); Chihara (1990) (pp. 148-149); Barwise (1975), which focuses on Kripke-Platek set theory with urelements, KPU; Barwise \& Moss (1996); Lewis (1991); Mendelson (2010) (§4.6.5); Jech (2002) (p. 250 ff.); Potter (2004); and Menzel (2014).
} 
From a purely technical perspective, three things make Potter's treatment unusual. First, Potter allows urelements in his basic axiomatization. This is quite handy for philosophical purposes, as it lets us talk about sets of individuals - people, atoms, space-time points, etc.-without employing awkward set-theoretic surrogates. (Bays (2005): paragraph 2.)

Likewise, Elliott Mendelson summarizes the need for such a system in terms of applicability:

The theories NBG, MK, ZF, NF, and ML do not allow for objects that are not sets or classes. This is all well and good for mathematicians, since only sets or classes seem to be needed for dealing with mathematical concepts and problems. However, if set theory is to be a part of a more inclusive theory having to do with the natural or social sciences, we must permit reference to things like electrons, molecules, people, companies, etc., and to sets and classes that contain such things. Things that are not sets or classes are sometimes called urelements. We shall sketch a theory UR similar to NBG that allows for the existence of urelements. (Mendelson (2010): 300-301)

Coming from a quite diverse range of philosophical angles, Field (1980), Quine (1986a), Steiner (1998), Potter (2004), Leng (2010) (and no doubt many others) agree with Mendelson. This is how Hartry Field puts it:

In order to be able to apply any postulated abstract entities to the physical world, we need impure abstract entities, e.g. functions that map physical objects to pure abstract entities. Such impure abstract entities serve as a bridge between pure abstract entities and the physical objects; without the bridge, the pure objects would be idle. Consequently, if we regard functions as sets of a certain sort, then the mathematical theories we should be considering must include at least a minimal amount of set theory with urelements (an urelement being a non-set which can be the member of sets). (Field (1980): 9)

If the mathematics to be applied is set theory, then it must be set theory with atoms.

\section{A Base Theory for Applied Mathematics}

Let ZF be standard Zermelo-Fraenkel set theory (including the axiom of choice); let restricted ZFU be ZF modified to allow for the existence of urelements, but not allowing for any non-set-theoretic vocabulary to appear in the comprehension axioms (for definiteness, we may stipulate that it contains as an axiom that there is a set of all non-sets); and if $V$ is a class of expressions, let $\mathrm{ZFU}_{V}$ be restricted ZFU together with any instances of the comprehension schemas in which the vocabulary in $V$ as well as the set-theoretic vocabulary is allowed to appear. What I earlier called 'full set theory' isn't really a single theory; rather, to 'apply full set theory' in the context of a theory $T$ is to apply $Z F U_{V(T)}$, where $V(T)$ is the vocabulary of $T$. (Field (1980): 17) 


\subsection{Introduction}

The approach we develop from here on is modeled on Field's summary quoted above. ${ }^{14}$ It is a many-sorted set theory, which I call Zermelo-Fraenkel set theory (with Choice) with atoms, over an application signature $\sigma$, denoted $\mathrm{ZFCA}_{\sigma} \cdot{ }^{15}$ However, it is worth pointing out that this is not very different from a formalism using type-theory or higher-order logic. ${ }^{16}$

\subsection{The Sort System}

We shall use a many-sorted formalization with four sorts: ${ }^{17}$

$\begin{array}{ll}\text { atom } & \text { Ranges over atoms. } \\ \text { bool } & \text { Ranges over booleans. } \\ \text { set } & \text { Ranges over sets. } \\ \text { global } & \text { Ranges over everything. }\end{array}$

One can, in fact, build a type hierarchy over these four sorts. I will define that in a moment, but will not really use it (except as a convenient mnemonic for declaring the types of various constants, predicates and function symbols). We are only interested in the first-order fragment. The sorts atom, bool and set are treated as disjoint, and all three are proper subsorts of global and, together, they exhaust global. ${ }^{18}$ There is one feature of the many-sorted logic worth stressing at the outset: this is not a conventional many-sorted system because (a) it includes subsorts (of the global sort) and (b) it is not an order-sorted system because "downwards substitution" is not always permitted over the global sort variables. ${ }^{19}$

We next define this a bit more precisely:

Definition 1 (Sorts). The expressions bool, atom, set and global are sorts. Let $S:=$ \{bool, atom, set, global\}.

14 An approach that seems to be similar is given in Mycielski (2013), where the base theory invoked is called $\mathrm{ZF}^{+}$and scientific theories are then treated as taking the form $\mathrm{ZF}^{+}+\Sigma$, where the scientific laws, etc., are elements of $\Sigma$.

15 The approach developed here is influenced by Jon Barwise's similar many-sorted formulation of KPU (Kripke-Platek set theory with urelements) in Barwise (1975). I am grateful to Ali Enayat for drawing my attention to Barwise's approach.

16 This would have been the preferred formalism of the logical empiricists, and especially Rudolf Carnap: e.g., Carnap (1928), Carnap (1966). See Leitgeb \& Carus (2020) for a detailed survey of Carnap's proposed formalization systems for science. Because of well-known intertranslatability results, these approaches may well be near notational equivalents, modulo some subtleties about proof-theoretic strength.

17 See Manzano (1996) for an exposition of many-sorted logic and its applications. Enderton (2001) also explains the main ideas. In his Quine (1956), Quine gives some reasons to prefer a singlesorted formalization of theories, although it's not clear how persuasive these reasons are given the intertranslatability of many-sorted and one-sorted theories.

18 In principle, we might introduce a richer system, perhaps with subsorts ord, real, natnum, etc., for sets, and with subsorts point, line, region, etc., for atoms.

19 For example, if $\mathrm{P}$ is a unary predicate on atoms, then $\mathrm{P}\left(a_{i}\right)$ is well-formed, for any atom variable $a_{i}$. But $\mathrm{P}\left(x_{i}\right)$ is also well-formed, where $x_{i}$ is a global variable. 
These expressions are not in the object language. ${ }^{20}$

Definition 2 (Type System). We define the full type system $S^{*}$ as follows. $S \subseteq S^{*}$. Furthermore, if $s_{1}, s_{2} \in S^{*}$, then $s_{1} \Rightarrow s_{2} \in S^{*}$. There are no other types. So the type system $S^{*}$ is defined by an inductive definition, using the sorts in $S$ as its base, and closing under the "constructor" $\Rightarrow .^{21}$

However, we shall not use the full type system, since we are only concerned with a first-order four-sorted formalism (wrt $S$ ), and not a full type hierarchy of variables for each type, along with constants, function symbols and predicates of various types.

Definition 3 (Sorted Variables). Corresponding to each sort is a special system of variables, defined as follows:

\begin{tabular}{lll} 
Sort & Variables & Range over \\
\hline atom & $a, a_{1}, a_{2}, \ldots$ & atoms. \\
bool & $\mathbf{b}, \mathbf{b}_{1}, \mathbf{b}_{2}, \ldots$ & truth values $\mathbb{T}$ and $\mathbb{F}$. \\
set & $X, X_{1}, X_{2}, \ldots$ & sets \\
global & $x, x_{1}, x_{2}, \ldots$ & objects
\end{tabular}

In general, each variable is assigned a sort as follows: ${ }^{22}$

$$
\begin{array}{ll}
\tau\left(a_{i}\right)=\text { atom } & \tau\left(\mathbf{b}_{i}\right)=\text { bool. } \\
\tau\left(X_{i}\right)=\text { set } & \tau\left(x_{i}\right)=\text { global. }
\end{array}
$$

Definition 4 (Type Declaration). A (first-order) type declaration for a symbol s has the form:

$$
\begin{array}{ll}
\text { (i) } & \mathrm{s}: \tau_{1} . \\
\text { (ii) } & \mathrm{s}: \tau_{1} \Rightarrow \cdots \Rightarrow \tau_{n} \Rightarrow \tau_{n+1} . \\
\text { (iii) } & \mathrm{s}: \tau_{1} \Rightarrow \cdots \Rightarrow \tau_{n} \Rightarrow \text { bool. }
\end{array}
$$

If the type of $\mathrm{s}$ is $\tau$ for some $\tau \in S$, then we say it is a (first-order) variable or constant. If the type of $\mathrm{s}$ is $\tau_{1} \Rightarrow \cdots \Rightarrow \tau_{n} \Rightarrow \tau_{n+1}$, then we say that $\mathrm{s}$ is a (first-order) $n$-ary function symbol. If the type of $\mathrm{s}$ is $\tau_{1} \Rightarrow \cdots \Rightarrow \tau_{n} \Rightarrow$ bool, then we say that $\mathrm{s}$ is a (first-order) $n$-ary predicate.

A remark is in order. I am using a version of Church type-theoretic notation (which is similar to Isabelle notation) as a mnemonic for the types of constants, predicates and function symbols that appear in the application signature. ${ }^{23}$ Nothing really hangs on this.

20 However, we shall show how to define them in the object language.

21 I have not said what this constructor $\Rightarrow$ "is". However it doesn't matter. I am using a type-theory notation here: " $s_{1} \Rightarrow s_{2}$ " means "the 'type' of functions from $s_{1}$ to $s_{2}$ ". Originally, Church (1940), who had two base sorts, $o$ (booleans) and $\iota$ (individuals), used a bracketing notation $(o o),(o \iota), o(o o)$, $(o \iota) \iota$, etc. (and read backwards: so $(o \iota)$ is $\iota \Rightarrow o$ ). See Coquand (2018) on type theory and Benzmüller \& Andrews (2019) for Church's type theory, for further explanation.

22 Thus, by " $\tau(s)=\ldots$.", we mean "the sort of symbol $s$ is . ..". The functional notation " $\tau(-)$ " belongs to the meta-language, not the object language.

23 Isabelle is a higher-order/type-theoretic theorem-proving system initially designed by Laurence Paulson. See Wenzel et al. (2020) for the user manual for Isabelle. 
Definition 5 (Application Signature). A (first-order) application signature $\sigma$ over the sorts $S$ is a (possibly empty) set of non-logical syntactic primitives

$$
\sigma=\overbrace{\left\{\mathrm{c}_{i}\right\}_{i \in I_{1}}}^{\text {constants }} \cup \overbrace{\left\{\mathrm{F}_{i}\right\}_{i \in I_{2}}}^{\text {function symbols }} \cup \overbrace{\left\{\mathrm{P}_{i}\right\}_{i \in I_{3}}}^{\text {predicates }}
$$

where each symbol has an associated type declaration as follows:

$$
\begin{array}{ll}
\mathrm{C} \text { is a constant } & \mathrm{C}: \tau(\mathrm{c}) \\
\mathrm{F} \text { is an } n \text {-ary function symbol } & \mathrm{F}: \tau_{1}(\mathrm{~F}) \Rightarrow \cdots \Rightarrow \tau_{n}(\mathrm{~F}) \Rightarrow \tau_{n+1}(\mathrm{~F}) . \\
\mathrm{P} \text { is an } n \text {-ary predicate symbol } & \mathrm{P}: \tau_{1}(\mathrm{P}) \Rightarrow \cdots \Rightarrow \tau_{n}(\mathrm{P}) \Rightarrow \text { bool. }
\end{array}
$$

where in each case, $\tau(\mathrm{c})$, the $\tau_{i}(\mathrm{~F})$ and the $\tau_{i}(\mathrm{P})$ are elements of $S$.

Signatures do not officially contain the identity predicate or the boolean constants. But we shall assume that every language built over a signature implicitly contains the binary identity predicate $=$ and the boolean constants.

Definition 6 (Boolean constants). Every language we consider will be assumed to contained the boolean constants $T$ and $\perp$, with type declarations:

$$
\top \text { : bool } \quad \perp \text { : bool }
$$

Definition 7 (Type Declaration for Identity). The predicate = has type declaration:

$$
=: \text { global } \Rightarrow \text { global } \Rightarrow \text { bool. }
$$

Identity is global, and thus $t_{1}=t_{2}$ is well-formed for any terms $t_{1}, t_{2}$.

Definition 8 (Purely Atomic). If a constant, predicate or function symbo has type declaration of the form:

$$
\begin{gathered}
\mathrm{c}: \text { atom } \\
\mathrm{P}: \text { atom } \Rightarrow \cdots \Rightarrow \text { atom } \Rightarrow \text { bool } \\
\mathrm{F}: \text { atom } \Rightarrow \cdots \Rightarrow \text { atom } \Rightarrow \text { atom }
\end{gathered}
$$

it is called "purely atomic".

A signature whose symbols are purely atomic is called a purely atomic signature. If $\sigma$ is a signature, then the restriction to its symbols which are purely atomic symbols is called its purely atomic subsignature, denoted $\sigma$ latom. If the component sorts for a function symbol are all the same, then I call the symbol homogeneous. If the component sorts vary, I call the symbol mixed. If the component sorts for a predicate symbol are all the same except for the compulsory final $\Rightarrow$ bool, then I call the predicate homogeneous. If the sorts vary, I call the predicate mixed.

Definition 9 (Auxiliary predicates). Let $\sigma$ be an application signature over $S$. For any $n$-ary function symbol $\mathrm{F}$ in $\sigma$, with type declaration,

$$
\mathrm{F}: \tau_{1}(\mathrm{~F}) \Rightarrow \cdots \Rightarrow \tau_{n}(\mathrm{~F}) \Rightarrow \tau_{n+1}(\mathrm{~F})
$$


we introduce an auxiliary $(n+1)$-ary predicate $\mathrm{P}_{\mathrm{F}}$, with type declaration:

$$
\mathrm{P}_{\mathrm{F}}: \tau_{1}(\mathrm{~F}) \Rightarrow \ldots \tau_{n}(\mathrm{~F}) \Rightarrow \tau_{n+1}(\mathrm{~F}) \Rightarrow \text { bool }
$$

Along with defining axiom:

$$
\left(\mathrm{Ax}_{\mathrm{F}}\right): \quad \mathrm{P}_{\mathrm{F}}\left(v_{1}, \ldots, v_{n}, v_{n+1}\right) \leftrightarrow v_{n+1}=\mathrm{F}\left(v_{1}, \ldots, v_{n}\right)
$$

where $v_{1}, \ldots, v_{n}$ are variables of the appropriate sorts.

From now on, whenever we introduce an application signature, we assume it has been extended with the corresponding auxiliary predicates.

Definition 10 (Extended signature: $\sigma_{\epsilon}$ ). Let $\sigma$ be an application signature over $S$. Let the extended signature $\sigma_{\in}:=\sigma \cup\{\in\}$ be the extension of $\sigma$ by adding the new binary predicate $\in$ with type declaration:

$$
\epsilon: \text { global } \Rightarrow \text { global } \Rightarrow \text { bool. }
$$

Note that $\in$ is global. Thus, $t_{1} \in t_{2}$ is a well-formed formula for any terms $t_{1}, t_{2}$.

\subsection{Unary Sort Predicates}

An advantage of a global variable sort, along with the three subsorts, is that we can "express the primitive sorts" inside the object language language itself:

Definition 11 (Unary Predicates for Sorts). For each primitive sort, we introduce a unary global predicate by explicit definition:

$$
\begin{aligned}
\operatorname{Dfn}_{\text {atom }}: & \forall x(\operatorname{atom}(x) \leftrightarrow \exists a(x=a)) . \\
\operatorname{Dfn}_{\text {bool }}: & \forall x(\operatorname{bool}(x) \leftrightarrow \exists \mathbf{b}(x=\mathbf{b})) . \\
\operatorname{Dfn}_{\text {set }}: & \forall x(\operatorname{set}(x) \leftrightarrow \exists X(x=X)) . \\
\operatorname{Dfn}_{\text {global }}: & \forall x\left(\operatorname{global}(x) \leftrightarrow \exists x_{1}\left(x=x_{1}\right)\right) .
\end{aligned}
$$

We let $D f n s$ be $\left\{D n_{\text {atom }}, D f n_{\text {bool }}, D f n_{\text {set }}, D f n_{\text {global }}\right\}$.

\subsection{Structures for $L\left(\sigma_{\epsilon}\right)$}

Definition 12. Let $\sigma=\left\{\mathrm{c}_{i}\right\}_{i \in I_{1}} \cup\left\{\mathrm{F}_{i}\right\}_{i \in I_{2}} \cup\left\{\mathrm{P}_{i}\right\}_{i \in I_{3}}$ be an application signature over $S$. A $\sigma_{\in}$-structure $M$ is a 4 -sorted (first-order) structure:

$$
M=\left(\text { global }^{M}, \text { set }^{M}, \text { atom }^{M}, \text { bool }^{M} ; \in^{M} ; \ldots,\left(\mathrm{c}_{i}\right)^{M}, \ldots,\left(\mathrm{F}_{i}\right)^{M}, \ldots,\left(\mathrm{P}_{i}\right)^{M}, \ldots\right)
$$

where the following ten conditions are satisfied: ${ }^{24}$

$\overline{24}$ Our structures interpret variables. So, if $v$ is a variable, then $v^{M}$ is an element of the domain. 
(1) global $^{M}$ is the global domain (values of global variables $x, x_{1}, \ldots$ ).

(2) set $^{M}$ is the collection of sets in $M$ (values of set variables $X, X_{1}, \ldots$ ).

(3) atom $^{M}$ is the collection of atoms in $M$ (values of atom variables $a, a_{1}, \ldots$ ).

(4) bool $^{M}=\{\mathbb{T}, \mathbb{F}\}$ (where $T^{M}=\mathbb{T} ; \perp^{M}=\mathbb{F}$ ).

(5) global $^{M}=$ set $^{M} \cup$ atom $^{M} \cup$ bool $^{M}$.

(6) global $^{M} \neq \varnothing ;$ set $^{M} \neq \varnothing$; atom ${ }^{M} \neq \varnothing$.

(7) set $^{M} \cap$ atom $^{M}=$ set $^{M} \cap$ bool $^{M}=$ atom $^{M} \cap$ bool $^{M}=\varnothing$.

(8) $\in^{M}$ is a subset of global $^{M} \times \operatorname{set}^{M}$ (the interpretation of $\in$ ).

And, for the variables, we have:

(9) $\left(\mathbf{b}_{i}\right)^{M} \in$ bool $^{M} ;\left(a_{i}\right)^{M} \in$ atom $^{M} ;\left(X_{i}\right)^{M} \in$ set $^{M} ;\left(x_{i}\right)^{M} \in$ global $^{M}$.

And, for each constant $\mathrm{c}$, function symbol $\mathrm{F}$ and predicate $\mathrm{P}$ of the non-logical symbols from $\sigma$, their interpretations $\mathrm{c}^{M}, \mathrm{~F}^{M}$ and $\mathrm{P}^{M}$ must respect the sorting built into $\sigma$ :

(10.a) $\mathrm{c}^{M} \in(\tau(\mathrm{c}))^{M}$.

(10.b) $\mathrm{F}^{M}:\left(\tau_{1}(\mathrm{~F})\right)^{M} \times \cdots \times\left(\tau_{n}(\mathrm{~F})\right)^{M} \rightarrow\left(\tau_{n+1}(\mathrm{~F})\right)^{M}$.

(10.c) $\mathrm{P}^{M} \subseteq\left(\tau_{1}(\mathrm{P})\right)^{M} \times \cdots \times\left(\tau_{n}(\mathrm{P})\right)^{M}$.

Various reducts might be defined. The most important is the single-sorted structure:

$$
M^{\text {atom }}:=\left(\text { atom }^{M} ; \ldots,\left(\mathrm{c}_{i}\right)^{M}, \ldots,\left(\mathrm{F}_{i}\right)^{M}, \ldots,\left(\mathrm{P}_{i}\right)^{M}, \ldots\right)
$$

where the $\mathrm{c}_{i}, \mathrm{~F}_{i}$ and $\mathrm{P}_{i}$ are purely atomic primitive symbols.

Identity is interpreted naturally, and the definitions of term denotation $t^{M}$ and satisfaction $M \models \phi$ are more or less straightforward.

\subsection{ZFCA ${ }_{\sigma}$ with sorts $\{$ bool, atom, set, global $\}$}

The remaining features of the sort system and syntax are relegated to the Appendix. ${ }^{25}$

Definition 13 (Quasi-logical axioms). The following $L\left(\sigma_{\in}\right)$-sentences are called quasilogical axioms (some of these are explained in the Appendix):

(1) For each function symbol $F$, the explicit definition $A x_{F}$ of its auxiliary predicate $P_{F}$. (Definition 9)

(2) Explicit definitions Dfns of predicates bool, atom, set and global. (Definition 11)

(3) The Boolean axioms (Definition 22).

(4) The Sort axioms (SA1), ., (SA5) (Definition 23)

(5) The Subsort axioms (Definition 24).

Lemma 1. Dfns $\vdash \forall a$ atom $(a) \wedge \forall \mathbf{b} \operatorname{bool}(\mathbf{b}) \wedge \forall X \operatorname{set}(X)$.

Proof. I show $\forall a$ atom $(a)$. The other conjuncts are similar. Suppose we have some $a$ with $\neg$ atom $(a)$. Hence, $\neg \exists a_{1}\left(a=a_{1}\right)$. So, for all $a_{1}$, we have $a \neq a_{1}$. In particular, $a \neq a$. Contradiction.

25 Definition 20 defines the well-formed terms and primitive formulas of $L\left(\sigma_{\epsilon}\right)$. Definition 21 completes the definition of the language $L\left(\sigma_{\epsilon}\right)$. 
One can prove from the "quasi-logical" axioms theorems expressing sort exclusion:

Lemma 2. Quasi-logical axioms $\vdash \forall a \forall X(a \neq X)$.

Proof. Suppose $\neg \forall a \forall X(a \neq X)$ and so, there is some $a$ and some $X$ such that $a=X$. Thus, atom $(a)$ and $\operatorname{set}(X)$ and $a=X$. So, $\exists x(x=a \wedge x=X \wedge$ atom $(x) \wedge \operatorname{set}(x))$. So, $\exists x(\operatorname{atom}(x) \wedge \operatorname{set}(x))$, which contradicts the sort axiom (SA1).

Definition 14 (Axioms of ZFCA ${ }_{\sigma}$ ). The non-logical axioms of $\mathrm{ZFCA}_{\sigma}$ in $L\left(\sigma_{\in}\right)$ are:

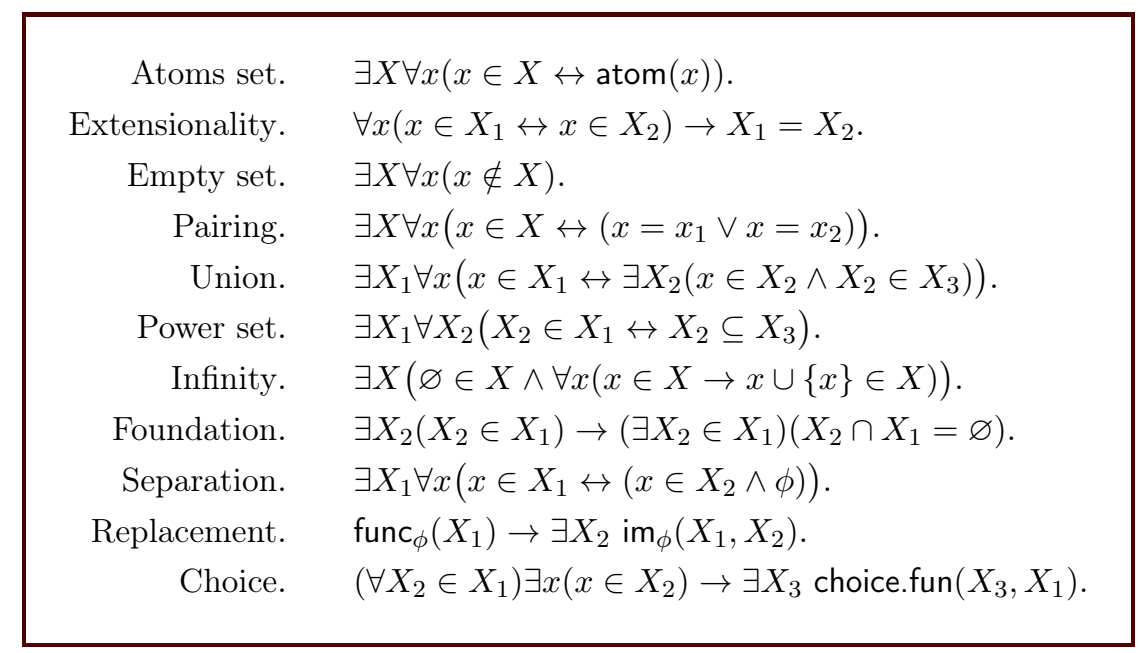

These axioms are all implicitly universally quantified. In instances of Separation, $\phi$ must not contain $X_{1}$ free. The axioms use the symbols " $\varnothing$ ", "ᄃ", " $\cap$ " and "\{.\}": we assume these are given their standard definitions.

The formulas $\phi$ appearing in the Separation and Replacement schemes belong to the full language $L\left(\sigma_{\epsilon}\right)$, and may contain parameters, including set parameters. ${ }^{26}$ In the formulation of Replacement, func ${ }_{\phi}\left(X_{1}\right)$ expresses that the formula $\phi$ defines a (class) function on (set) $X_{1}$. And $\operatorname{im}_{\phi}\left(X_{1}, X_{2}\right)$ expresses that $X_{2}$ is the image of $X_{1}$ under the (class) function defined by $\phi$. In the formulation of Choice, choice.fun $\left(X_{3}, X_{1}\right)$ expresses that $X_{3}$ is a choice function on $X_{1}$.

In addition, we have the quasi-logical axioms of $\mathrm{ZFCA}_{\sigma}$. So long as sort restrictions are not violated (e.g., in quantifier instantiations and in substitutions), the deductive system is a fairly straightforward modification of that for 1 -sorted first-order logic. ${ }^{27}$

I will sometimes call ZFCA $\sigma$ the standard base theory for applied mathematics (with respect to the signature $\sigma$ over the sort system $S$ ).

${ }_{26}$ In principle, one could weaken the theory by requiring that $\phi$ may only belong to the sublanguage $L(\sigma$ latom $)$ - the result would be a kind of predicative weakening.

27 The most important modification is this. Standardly in many-sorted logic, the logical axiom $\forall v \phi(v) \rightarrow$ $\phi_{t}^{v}$ requires the term $t$ substituted for $v$ to have same sort as that of $v$ (in order-sorted MSL, the sort of $t$ may be a subsort of that of $v$ ). In our system, we also have global variables, and in general, an additional logical theorem $\forall x \phi(x) \rightarrow \phi_{t}^{x}$, where the sort of $t$ may be of any sort subject to $\phi_{t}^{x}$ being well-formed. This is handled by the additional quasi-logical subsort axioms specified in Definition 24. 
Regarding its properties, note first that $\mathrm{ZFCA}_{\sigma}$ implies the existence of just one atom, since $\exists a(a=a)$ is theorem of logic. Although there is a set of atoms, ZFCA $\sigma$ can be shown to be neutral on there being more than one element of this set. Note second that $\mathrm{ZFCA}_{\sigma}$ is powerful enough to model all known mathematics, and certainly all core mathematics. Third, note that there is some redundancy here: Replacement implies Separation (and Separation implies Empty Set) and (assuming Empty set and Power set) Pairing. Notationally, $Z_{F}$ is $Z_{F C A}$ without Choice; $Z_{\sigma}$ is $Z_{F} A_{\sigma}$ without Replacement; $\mathrm{ZFA}_{\sigma}^{-\infty}$ is $\mathrm{ZFA}_{\sigma}$ without Infinity; $\mathrm{ZA}_{\sigma}^{-\infty}$ is $\mathrm{ZA}_{\sigma}$ without Infinity. For many examples, finitary set theory (with atoms) $\mathrm{ZA}_{\sigma}^{-\infty}$ is perfectly adequate.

Fourth, there is a standard technique for translating a many-sorted theory into a one-sorted equivalent: corresponding to each sort $s$, one introduces a unary one-sorted predicate $P_{s}(x)$. The translation ${ }^{\circ}: L_{\text {many-sorted }} \rightarrow L_{\text {one-sorted }}$ then takes, e.g., a formula $\forall v \phi(v)$, where $v$ has sort $s$, to a relativized one-sorted formula $\forall x\left(P_{s}(x) \rightarrow \phi^{\circ}(x)\right) .{ }^{28}$ Here, essentially because it uses global variables and each sort atom, ... has a corresponding unary sort predicate atom $(x), \ldots$, the system $\mathrm{ZFCA}_{\sigma}$ in fact contains that translation as a subtheory (a subtlety about the translation of function symbols is dealt with via the auxiliary predicates introduced in Definition 9).

I will henceforth assume that ZFCA $\sigma$ also contains the various explicit definitions needed to develop and formalize all core mathematics. Strictly speaking, what we are calling $\mathrm{ZFCA}_{\sigma}$ is a conservative extension of $\mathrm{ZFCA}_{\sigma}$ given by a complicated series of explicit definitions. ${ }^{29}$

Definition 15. "Atoms set" asserts there is a set $X$ of all atoms. We have uniqueness by Extensionality. So we can define a set constant (i.e., of sort set) $\mathbb{A}$ such that

$$
\forall x(x \in \mathbb{A} \leftrightarrow \operatorname{atom}(x))
$$

In this many-sorted system, we no longer need a separate axiom that "atoms lack elements". It is provable from the set of quasi-logical axioms:

Lemma 3. Quasi-logical axioms $\vdash \forall a \forall x(x \notin a)$.

Proof. The sort axiom (SA5) for $\in$ states: $\forall x_{1} \forall x_{2}\left(x_{1} \in x_{2} \rightarrow \operatorname{set}\left(x_{2}\right)\right)$. Using the definition ( $\left(\mathrm{Dfn}_{\text {set }}\right)$, we infer: $\forall x_{1} \forall x_{2}\left(x_{1} \in x_{2} \rightarrow \exists X\left(x_{2}=X\right)\right)$. Thus, by a Subsort axiom, $x_{1} \in a \rightarrow \exists X(a=X)$. But by Lemma $2, \forall X(a \neq X)$ and so $\neg \exists X(a=X)$. So, $x_{1} \notin a$. And since $a, x_{1}$ are arbitrary, generalization gives: $\forall a \forall x(x \notin a)$.

\section{Example 3. Defining impure sets}

Consider Pairing: $\exists X \forall x\left(x \in X \leftrightarrow\left(x=x_{1} \vee x=x_{2}\right)\right)$. Suppose we wish to assert the existence of an impure set: say, defined by the formula $x=\mathrm{c} \vee x=\varnothing$, where $\mathrm{c}$ is a constant denoting an atom: i.e., the existence of $X=\{c, \varnothing\}$. This is given by the instance:

$$
\exists X \forall x(x \in X \leftrightarrow(x=\mathrm{c} \vee x=\varnothing))
$$

\footnotetext{
28 The translation was first given in Schmidt (1938), and later in more detail, Wang (1952).

29 E.g., " $X$ is the ordered pair of $x_{1}$ and $x_{2}$ ", " $X_{1}$ is a binary relation on $X_{2}$ ", " $X$ is a real number", etc.
} 
Formulating this in 2-sorted logic without global variables seems to be tricky. But the use of global variables simplifies it enormously.

Definition 16. Consider Pairing again. An instance is:

$$
\exists X \forall x(x \in X \leftrightarrow(x=\top \vee x=\perp))
$$

We have uniqueness by Extensionality. So, define a set constant $\mathbb{B}$ such that

$$
\forall x(x \in \mathbb{B} \leftrightarrow \operatorname{bool}(x))
$$

\section{Comprehension and Set Abstracts}

For set theorists, the interest of ZFA is connected to permutation models and independence proofs. Because no structure whatsoever is imposed on the atoms, the atoms are, in a sense, "indiscernible" objects, compared with the sets. However, for us, the situation is different, because the scientific axioms written in the application language $L(\sigma)$ will typically impose structure onto the atoms - the atoms may be asserted to be structured as an ordering, a geometry, a discrete structure, a graph, etc. Consequently, such methods don't transfer; or at least not easily.

\subsection{Comprehension}

Definition 17. Let $\phi\left(a_{1}, \ldots, a_{n}\right)$ be an $L\left(\sigma_{\epsilon}\right)$-formula containing only atom variables free. We say that $\phi$ expresses a relation on atoms.

Even if $\phi$ expresses a relation on atoms, this is compatible with $\phi$ containing further expressions referring to non-atoms. For example, suppose $\ell_{\mathrm{m}}$ is a mixed function symbol with type declaration atom $\Rightarrow$ set. Perhaps $\ell_{\mathrm{m}}(a)$ can be read to mean: "the length in metres of concrete atom $a$ ". Even so, the following formula does satisfy that condition:

$$
\phi\left(a_{1}, a_{2}\right):=\left(\ell_{\mathrm{m}}\left(a_{2}\right)=\pi \cdot \ell_{\mathrm{m}}\left(a_{1}\right)\right)
$$

Indeed, $\phi$ might express a relation on atoms which is not definable in the purely atomic fragment, $L(\sigma$ 「atom $)$.

Lemma 4. Suppose $\phi\left(a_{1}, \ldots, a_{n}\right)$ expresses a relation on atoms. Then:

$$
\mathrm{ZA}_{\sigma}^{-\infty} \vdash \exists X \forall a_{1} \ldots \forall a_{n}\left(\left(a_{1}, \ldots, a_{n}\right) \in X \leftrightarrow \phi\left(a_{1}, \ldots, a_{n}\right)\right) .
$$

Proof. By separation, we have: $\exists X_{1} \forall x\left(x \in X_{1} \leftrightarrow\left(x \in X_{2} \wedge \psi(x)\right)\right)$. Thus, letting $\psi(x)$ be $\exists a_{1} \ldots \exists a_{n}\left(x=\left(a_{1}, \ldots a_{n}\right) \wedge \phi\left(a_{1}, \ldots a_{n}\right)\right)$, we get:

$$
\exists X_{1} \forall x\left(x \in X_{1} \leftrightarrow\left[x \in X_{2} \wedge \exists a_{1} \ldots \exists a_{n}\left(x=\left(a_{1}, \ldots a_{n}\right) \wedge \phi\left(a_{1}, \ldots a_{n}\right)\right)\right]\right) .
$$

By instantiating $X_{2}$ with the set term $\mathbb{A}^{n}$, this yields that there is a set $X_{1}$ st:

$$
\forall x\left(x \in X_{1} \leftrightarrow\left[x \in \mathbb{A}^{n} \wedge \exists a_{1} \ldots \exists a_{n}\left(x=\left(a_{1}, \ldots a_{n}\right) \wedge \phi\left(a_{1}, \ldots a_{n}\right)\right)\right]\right) .
$$


Let us assume: $\left(a_{n+1}, \ldots, a_{2 n}\right) \in X_{1}$. By (23), we infer $\exists a_{1} \ldots \exists a_{n}\left(\left(a_{n+1}, \ldots, a_{2 n}\right)=\right.$ $\left.\left(a_{1}, \ldots a_{n}\right) \wedge \phi\left(a_{1}, \ldots a_{n}\right)\right)$. And so (using the properties of ordered $n$-tuples), $\phi\left(a_{n+1}, \ldots, a_{2 n}\right)$, as claimed. Conversely, this reasoning runs backwards too. Suppose $\phi\left(a_{n+1}, \ldots, a_{2 n}\right)$. By Lemma 1 , we have atom $\left(a_{n+1}\right), \ldots$, atom $\left(a_{2 n}\right)$. This gives $a_{n+1} \in \mathbb{A}, \ldots, a_{2 n} \in \mathbb{A}$. And so, $\left(a_{n+1}, \ldots, a_{2 n}\right) \in \mathbb{A}^{n}$. Furthermore, we have $\exists a_{1} \ldots \exists a_{n}\left(\left(a_{n+1}, \ldots, a_{2 n}\right)=\left(a_{1}, \ldots a_{n}\right) \wedge \phi\right)$. Thus, from $(23),\left(a_{n+1}, \ldots, a_{2 n}\right) \in X_{1}$.

So, conjoining these two conclusions, and relabelling variables:

$$
\exists X_{1} \forall a_{1} \ldots \forall a_{n}\left(\left(a_{1}, \ldots, a_{n}\right) \in X_{1} \leftrightarrow \phi\left(a_{1}, \ldots, a_{n}\right)\right)
$$

Lemma 5. Let $\mathrm{P}$ be a purely atomic $n$-ary predicate from $\sigma$. Then

$$
\mathrm{ZA}_{\sigma}^{-\infty} \vdash \exists X \forall a_{1} \ldots \forall a_{n}\left(\left(a_{1}, \ldots, a_{n}\right) \in X \leftrightarrow \mathrm{P}\left(a_{1}, \ldots, a_{n}\right)\right) .
$$

Proof. $\mathrm{P}\left(a_{1}, \ldots, a_{n}\right)$ expresses a relation on atoms.

\subsection{Set Abstracts}

Lemma 4 and Lemma 5 imply that, under certain conditions, we can introduce an explicit definition of a set constant (aka set abstract):

$$
\begin{aligned}
& \bar{\phi}:=\left\{\left(a_{1}, \ldots, a_{n}\right) \mid \phi\left(a_{1}, \ldots, a_{n}\right)\right\} \\
& \overline{\mathrm{P}}:=\left\{\left(a_{1}, \ldots, a_{n}\right) \mid \mathrm{P}\left(a_{1}, \ldots, a_{n}\right)\right\}
\end{aligned}
$$

satisfying a corresponding condition:

$$
\begin{aligned}
& \left(a_{1}, \ldots, a_{n}\right) \in \bar{\phi} \leftrightarrow \phi\left(a_{1}, \ldots, a_{n}\right) \\
& \left(a_{1}, \ldots, a_{n}\right) \in \overline{\mathrm{P}} \leftrightarrow \phi\left(a_{1}, \ldots, a_{n}\right)
\end{aligned}
$$

Loosely speaking, comprehension permits us to "convert" the predicates into constants, and then we may reason accordingly about the impure mathematical objects denoted by these constants. These objects are themselves impure sets/relations. For example, the constant $\mathbb{A}$ refers to the set of atoms and the constant $\bar{P}$ refers to an impure $n$-ary relation on atoms. And because the applied mathematics is geared up to prove general claims about sets and relations (irrespective of their underlying domain), there is an interplay between such general claims and their instantiations onto constants for impure objects like (the denotations of) $\mathbb{A}, \bar{P}$, etc.

\subsection{Set Abstracts for Mixed Symbols}

A similar point will apply to mixed primitives. However, one has to be a bit careful to ensure that the set variables are bound in a certain way, and also about dealing with function symbols defined only on a special subsort. Let us suppose the application signature contains a unary mixed function symbol $\mathrm{F}$ with type declaration:

$$
\mathrm{F}: \text { atom } \rightarrow \text { set }
$$


There are two reasons we cannot "lift" this to a constant $\bar{F}$ denoting its extension (i.e., graph). The only way to do so is to apply separation: ${ }^{30}$

$$
\exists X_{1} \forall x_{1} \forall x_{2}\left(\left(x_{1}, x_{2}\right) \in X_{1} \leftrightarrow\left(\left(x_{1}, x_{2}\right) \in X_{2} \wedge \phi\left(x_{1}, x_{2}\right)\right)\right)
$$

However, there is no set of sets. So I cannot as yet instantiate the set variable $X_{2}$ on a suitable "container set". Second, the relevant formula $\phi$ would be $x_{2}=\mathrm{F}\left(x_{1}\right)$. But this is not well-formed, since the well-formedness of $\mathrm{F}(t)$ requires that $t$ be of sort atom. The solution to the first problem involves the auxiliary predicate $P_{F}$ (Definition 9). In this case, it has type declaration

$$
\mathrm{P}_{\mathrm{F}}: \text { atom } \Rightarrow \text { set } \Rightarrow \text { bool }
$$

and defining axiom:

$$
\mathrm{P}_{\mathrm{F}}(a, X) \leftrightarrow X=\mathrm{F}(a)
$$

Since $\mathrm{P}_{\mathrm{F}}\left(x_{1}, x_{2}\right)$ is well-formed, using global variables, I can now form the required separation axiom:

$$
\exists X_{1} \forall x_{1} \forall x_{2}\left(\left(x_{1}, x_{2}\right) \in X_{1} \leftrightarrow\left(\left(x_{1}, x_{2}\right) \in X_{2} \wedge \mathrm{P}_{\mathrm{F}}\left(x_{1}, x_{2}\right)\right)\right)
$$

which is now well-formed. I am however left with the problem that I need a "container" set $X_{2}$ to make this work.

What this container set will be will vary from case to case, depending on what $F$ means in the specific application. Often, function symbols in physical laws map to the reals; sometimes to a structure built over the reals; sometimes to some vector space, or perhaps Lie group. There is no difficulty in defining set terms for these, and therefore as terms for bona fide sets: e.g., $\mathbb{R}, \mathbb{R}^{n}, C^{\infty}\left[\mathbb{R}^{n}\right], L^{2}\left[\mathbb{R}^{n}\right], \ldots$ In these cases, one then includes a special axiom for $\mathrm{F}$, stating its codomain as a set, thus bounding its values. This resolves the whole problem. For example, perhaps $F$ formalizes a quantity with real-values, like a scalar field. Its codomain axiom is then:

$$
\forall a(\mathrm{~F}(a) \in \mathbb{R})
$$

The product $\mathbb{A} \times \mathbb{R}$ is now our required "container set". Using Separation with respect to $\mathbb{A} \times \mathbb{R}$, on the auxiliary predicate:

$$
\exists X_{1} \forall x_{1} \forall x_{2}\left(\left(x_{1}, x_{2}\right) \in X_{1} \leftrightarrow\left(\left(x_{1}, x_{2}\right) \in \mathbb{A} \times \mathbb{R} \wedge \mathrm{P}_{\mathrm{F}}\left(x_{1}, x_{2}\right)\right)\right)
$$

Since this $X_{1}$ is unique by Extensionality, we may next explicitly define:

$$
\overline{\mathrm{F}}:=\left\{\left(x_{1}, x_{2}\right) \in \mathbb{A} \times \mathbb{R} \mid \mathrm{P}_{\mathrm{F}}\left(x_{1}, x_{2}\right)\right\}
$$

\footnotetext{
30 This form of separation, for relations, is equivalent to the original form: $\exists X_{1} \forall x_{3}\left(x_{3} \in X_{1} \leftrightarrow\right.$ $\left.\exists x_{1} \exists x_{2}\left(x_{3}=\left(x_{1}, x_{2}\right) \in X_{2} \wedge \phi\left(x_{1}, x_{2}\right)\right)\right)$ if we have Pairing.
} 
This somewhat roundabout method provides for the explicit definition:

$$
\overline{\mathrm{F}}:=\{(a, X) \in \mathbb{A} \times \mathbb{R} \mid X=\mathrm{F}(a)\}
$$

And from this, we then have the usual comprehension equivalence:

$$
(a, X) \in \overline{\mathrm{F}} \leftrightarrow X=\mathrm{F}(a)
$$

The point generalizes to other mixed primitives, both predicates and function symbols.

\section{Abstract Counterparts: Equivalence Modulo Mathemat- ics}

... let us abbreviate the statement "the set of planets belongs to the number nine" as $P$, and the statement "there is an $x$ and there is a $y$ and $\ldots$ such that $x$ is a planet and $y$ is a planet and ... and $x \neq y$ and ... and such that for every $z$, if $z$ is a planet then $z=x$ or $z=y$ or ...", which expresses "the number of planets is nine" in a purely first order way, as $P^{*}$. The equivalence $P \leftrightarrow P^{*}$ is a theorem of Principia, and hence holds in all models. (Putnam (1967): 31).

An important part of understanding how applied mathematics works concerns the relation between certain kinds of non-mathematical statement and certain mathematical statements which are, in some sense, "equivalent". Such equivalences in the context of applied mathematics were, so far as I know, first explicitly noted in the quote from Putnam above.

Suppose we have the following sentences,

$$
\begin{array}{r}
\exists_{9} x \text { planet }(x) \\
\text { \#planet }=9
\end{array}
$$

where $\exists_{9} x$ planet $(x)$ is formulated using numerically definite quantifiers $\exists_{n}$. Letting PM stand for Principia Mathematica, Putnam's point is:

$$
\mathrm{PM} \vdash \exists_{9} x \text { planet }(x) \leftrightarrow(\# \overline{\text { planet }}=9)
$$

Building on Putnam's work, Field (1980) suggested we might consider mathematical statements like "\#planet $=9$ " to be abstract counterparts of non-mathematical statements like " $\exists_{9} x$ planet $(x)$ ". Under Quine's analysis of ontological commitment, (37) carries a commitment only to planets. On the other hand, the second (38) is a mathematical claim, implying there is set with a certain cardinality.

In the context of the base theory we are using, suppose the signature $\sigma$ contains a unary predicate planet on atoms. Then:

$$
\mathrm{ZA}_{\sigma}^{-\infty} \vdash \exists_{9} a \text { planet }(a) \leftrightarrow(\# \overline{\text { planet }}=9)
$$


So: (37) and (38) are equivalent, modulo $\mathrm{ZA}_{\sigma}^{-\infty}$ (infinity is not needed for such simple equivalences). Another mathematical equivalence, modulo $\mathrm{ZA}_{\sigma}^{-\infty}$, is:

$$
\mathrm{ZA}_{\sigma}^{-\infty} \vdash \forall a(\operatorname{rabbit}(a) \rightarrow \operatorname{mammal}(a)) \leftrightarrow(\overline{\mathrm{rabbit}} \subseteq \overline{\text { mammal }})
$$

The relevant meta-theorems have the generic form:

\section{AbStract Equivalence}

$$
\mathrm{ZFCA}_{\sigma} \vdash \phi \leftrightarrow \phi^{\text {abs }}
$$

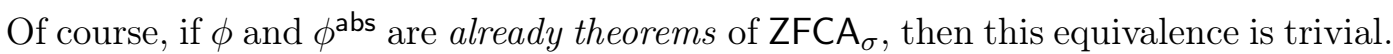
However, all the interesting cases for applied mathematics occur when $\phi$ and $\phi^{\text {abs }}$ are emphatically not theorems of $\mathrm{ZA}_{\sigma}, \mathrm{ZFA}_{\sigma}, \mathrm{ZFCA}_{\sigma}$, etc. ${ }^{31}$ In the next section, I will give several kinds of example of such abstract equivalences, modulo set theory.

\section{$6 \quad$ Systems and Equivalences}

\subsection{Suppes Equivalence}

Fix a signature $\sigma=\left\{\mathrm{P}_{1}, \mathrm{P}_{2}\right\}$ with type declarations $\mathrm{P}_{1}$ : atom $\Rightarrow$ bool and $\mathrm{P}_{2}$ : atom $\Rightarrow$ bool. Let a very simple law $\Theta \in L(\sigma)$ be given:

$$
\Theta\left(\mathrm{P}_{1}, \mathrm{P}_{2}\right): \quad \forall a\left(\mathrm{P}_{1}(a) \rightarrow \mathrm{P}_{2}(a)\right)
$$

We next define the corresponding (binary) "structural predicate" for $\Theta$ :

$$
\Theta_{s}\left(X_{1}, X_{2}\right): \quad \forall a\left(a \in X_{1} \rightarrow a \in X_{2}\right)
$$

And then we define the (unary) "Suppes predicate":32

$$
\bmod _{\Theta}\left(X_{3}\right): \quad \exists X_{1} \exists X_{2}\left(X_{3}=\left(X_{1}, X_{2}\right) \wedge \Theta_{s}\left(X_{1}, X_{2}\right)\right)
$$

Inside $\mathrm{ZA}_{\sigma}^{-\infty}$, using separation and pairing we can certainly prove the existence of the structure $\left(\overline{P_{1}}, \overline{P_{2}}\right)$. So, consider the sentence, which I call the Suppes sentence for $\Theta$ :

$$
\operatorname{Supp}(\Theta): \quad \bmod _{\Theta}\left(\left(\overline{P_{1}}, \overline{P_{2}}\right)\right)
$$

It is easy to see that the $\operatorname{Supp}(\Theta)$ is equivalent to $\Theta$ in (quite weak) set theory.

31 Analogously, Choice is equivalent to various other statements, using, say, Z set theory as a background base theory. Similarly, within the Reverse Mathematics programme of proving the equivalence of certain second-order set existence axioms with certain mathematical statements, using weaker subsystems as a base theory. For example, many interesting claims in analysis are equivalent to $\mathrm{ACA}_{0}$,

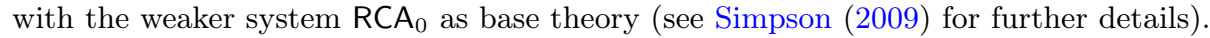

32 This term seems to originate in da Costa \& Chuaqui (1988). The slogan often repeated by Patrick Suppes is: "To axiomatize a theory is to define a predicate in terms of notions of set theory. A predicate so defined is called a set-theoretical predicate" (Suppes (1957), Ch.12 §12.2). 
Lemma 6. $\mathrm{ZA}_{\sigma}^{-\infty} \vdash \Theta \leftrightarrow \bmod _{\Theta}\left(\left(\overline{\mathrm{P}_{1}}, \overline{\mathrm{P}_{2}}\right)\right)$.

Proof. Consider the set abstracts $\overline{\mathrm{P}_{1}}$ and $\overline{\mathrm{P}_{2}}$, which satisfy:

$$
\forall x\left(x \in \overline{\mathrm{P}_{1}} \leftrightarrow \mathrm{P}_{1}(x)\right) \quad \forall x\left(x \in \overline{\mathrm{P}_{2}} \leftrightarrow \mathrm{P}_{2}(x)\right)
$$

Substituting $\overline{\mathrm{P}_{1}}$ for $X_{1}$, and $\overline{\mathrm{P}_{2}}$ for $X_{2}$, in the structural predicate $\Theta_{s}\left(X_{1}, X_{2}\right)$, we obtain: $\Theta_{s}\left(X_{1} / \overline{\mathrm{P}_{1}}, X_{2} / \overline{\mathrm{P}_{2}}\right) \leftrightarrow \forall a\left(a \in \overline{\mathrm{P}_{1}} \rightarrow a \in \overline{\mathrm{P}_{2}}\right)$. Using the equivalences above, we infer:

$$
\Theta_{s}\left(X_{1} / \overline{\mathrm{P}_{1}}, X_{2} / \overline{\mathrm{P}_{2}}\right) \leftrightarrow \Theta .
$$

Next, consider $\bmod _{\Theta}\left(\left(\overline{\mathrm{P}_{1}}, \overline{\mathrm{P}_{2}}\right)\right)$. This is equivalent to $\exists X_{1} \exists X_{2}\left(\left(\overline{\mathrm{P}_{1}}, \overline{\mathrm{P}_{2}}\right)=\left(X_{1}, X_{2}\right) \wedge\right.$ $\left.\Theta_{s}\left(X_{1}, X_{2}\right)\right)$. But, assuming pairing, this is equivalent to $\Theta_{s}\left(X_{1} / \overline{\mathrm{P}_{1}}, X_{2} / \overline{\mathrm{P}_{2}}\right)$. So,

$$
\bmod _{\Theta}\left(\left(\overline{\mathrm{P}_{1}}, \overline{\mathrm{P}_{2}}\right)\right) \leftrightarrow \Theta_{s}\left(X_{1} / \overline{\mathrm{P}_{1}}, X_{2} / \overline{\mathrm{P}_{2}}\right)
$$

Combining (48) and (49) we obtain the claim:

$$
\Theta \leftrightarrow \bmod _{\Theta}\left(\left(\overline{P_{1}}, \overline{P_{2}}\right)\right)
$$

Generalizing, it is not difficult to see that, modulo set theory with atoms, we can prove an equivalence between a (finitely axiomatized) theory $\Theta$ (in $L(\sigma)$, where $\sigma$ is purely atomic) and its Suppes sentence: ${ }^{33}$

$$
\overbrace{\Theta\left(\mathrm{P}_{1}, \ldots, \mathrm{P}_{n}\right)}^{\text {Theory }} \leftrightarrow \overbrace{\bmod _{\Theta}\left(\left(\overline{\mathrm{P}_{1}}, \ldots, \overline{\mathrm{P}_{n}}\right)\right)}^{\text {Suppes sentence of theory }}
$$

In general, there is class of theories $\Theta$ which are equivalent, modulo set theory with atoms, to their Suppes sentence: ${ }^{34}$

SUPPES EQUIVALENCE

$$
\mathrm{ZA}_{\sigma} \vdash \overbrace{\Theta}^{\text {Theory }} \leftrightarrow \overbrace{\bmod _{\Theta}(P)}^{\text {Suppes sentence Supp }(\Theta)}
$$

33 One might wish to bring all the "models" together, as a set. In general, this is not possible, for class/set reasons. However, it is possible to form the set of models which all live inside a suitably large "container set", called a universe. In Muller (2011), the container set or universe is a von Neumann universe $V_{\omega+n}$, for some largish $n$. In fact, to ensure we get the right equivalence for set theory with atoms, it would need to be $V_{\omega+n}(A)$, where $A$ is the set of atoms.

34 If $\sigma$ is purely atomic, this is true for all finitely axiomatized theories in $L(\sigma)$. In the broader case, it remains true for certain finitely axiomatized theories in $L\left(\sigma_{\epsilon}\right)$, but one must be more careful about the set quantifiers appearing in the axioms of $\Theta$ : these quantifiers need to be bounded in some way, so that they range over a set. In such cases, Suppes Equivalence holds too. 


\subsection{Remark on The "Semantic" vs "Syntactic" View Debate}

It's worth remarking here that the phenomenon I've called Suppes Equivalence may provide a constructive way of looking at the long-standing debate in the philosophy of science concerning "what theories are". This is quite a complicated debate, so I simplify. ${ }^{35}$

On one side are those who take the standard logical view: a theory is a set of sentences (or propositions expressed by sentences), which might in principle be formalized. I shall call these propositionalists. ${ }^{36}$ On the other side are those who say that a theory is a "collection of models" or "collection of structures". I shall call these structuralists. ${ }^{37}$

Certainly, it has been argued before these approaches are, somehow, two ways of putting the same thing (e.g., Worrall (1984), Friedman (1982)) ${ }^{38}$ Roughly, the argument notes that, given a theory $\Theta$ in $L(\sigma)$, one may define its model class, $\operatorname{Mod}_{\sigma}(\Theta)$, which is a class of structures (of signature $\sigma$ ). And given a class $\Sigma$ of structures of uniform signature $\sigma$, one may define its theory, $T h_{\sigma}(\Sigma)$ : the set of all sentences (in the signature $\sigma$ of the structures) which are true in all the structures. In some cases, specifying the set of (uninterpreted) sentences and specifying the model class will be equivalent. However, while one can indeed do this, these operations are not mutual inverses, except in highly constrained circumstances.

That said, one can give another argument, which is based on two points following from the previous discussion. First, an objection to the structuralist claim that "a theory is a collection of structures" is that this conception of what a theory is makes it impossible for a theory to be true. ${ }^{39}$ But surely theories are truth bearers. ${ }^{40}$ There is, however, a

35 One can simplify a bit too much as well. Steven French has published a new book, French (2020), There Are No Such Things As Theories, claiming theories don't exist.

36 Such a view was standard amongst the logical empiricists, and that remained so to the 1960s. For example, Carnap (1928), Carnap (1966), Hempel (1952), Przełęcki (1969) and others. It is sometimes called "the received view", or "the syntactic view of theories".

37 It is sometimes called "the semantic view of theories" or "the model-theoretic view". The beginnings of this view are usually traced to articles McKinsey et al. (1953), Suppes (1960), a couple more by Patrick Suppes et al, Suppes's textbook Suppes (1957) (see Ch. 12, §12.2: "Set-Theoretical Predicates and the Axiomatization of Theories"), and then followed by important and influential developments in Sneed (1971), Suppe (1977) and van Fraassen (1980). Joseph Sneed's work came to influence the "Munich school": e.g., Stegmüller (1976), Balzer et al. (1987). A valuable and sympathetic summary of the history of the semantic or model-theoretic or structuralist view is Muller (2011).

38 A discussion arguing that the alleged flaws are misrepresentations is Lutz (2012). Further criticisms of the semantic view have appeared in Halvorson (2012), Halvorson (2013) and Halvorson (2016), as well as Lutz (2014) and Lutz (2017).

39 I might add that the first objection, the Friedman-Worrall objection, seems to me not quite relevant. Like any other logician, I present, for example, Peano arithmetic PA as a theory in $L\left(\sigma_{\text {arith }}\right)$, where $\sigma_{\text {arith }}=\{0, s,+, \times\}$. PA has six axioms PA $1, \ldots, \mathrm{PA} 6$ and an induction scheme $\operatorname{Ind}\left(L\left(\sigma_{\text {arith }}\right)\right)$. Obviously, I may consider $\operatorname{Mod}_{\sigma_{\text {arith }}}(\mathrm{PA})$. For example, Kaye (1991) is a book about these models of PA. However, this excludes the crucial additional point that PA is true. That is, PA is a formalized theory of number theory - an interpreted theory in an interpreted language, with a fixed, definite intended interpretation: $(\mathbb{N}, 0, S,+, \times)$. In saying PA is true, I mean that each axiom of PA is true in $(\mathbb{N}, 0, S,+, \times)$.

40 I call this the Truth Bearer Objection. I've occasionally presented it in talks since the 1990s. 
response. This is that there is indeed a truth bearer: namely, what the structuralists call the "theoretical hypothesis".

So one now has a theory formulation favoured by structuralists. Moreover, we can, in a sense, internalize the theory formulation favoured by structuralists inside the applied mathematics base theory. For Suppes Equivalence tells us that a theory $\Theta$ as originally formulated in $L\left(\sigma_{\in}\right)$ is equivalent, in the base theory to the corresponding Suppes sentence $\operatorname{Supp}(\Theta)$. But the Suppes sentence just is what the structuralists call the "theoretical hypothesis". ${ }^{41}$ So, the propositionalist formulation of a theory and the structuralist formulation of the same theory are equivalent.

For example, take a simple quantitative law:

$$
\text { (L) For all time instants } t, F(t)=0 \text {. }
$$

To put this into our formalization, we fix a signature $\sigma=\{$ tim.inst, $\mathrm{F}\}$ with type declarations tim.inst : atom $\Rightarrow$ bool and $F$ : atom $\Rightarrow$ set. We shall have two axioms formulating our quantitative law (I call the first the codomain axiom for the quantity):

$$
\begin{gathered}
\forall a(\mathrm{~F}(a) \in \mathbb{R}) \\
\forall a(\operatorname{tim} \cdot \operatorname{inst}(a) \rightarrow \mathrm{F}(a)=0)
\end{gathered}
$$

where $\mathbb{R}$ and 0 are defined constants in $L\left(\sigma_{\epsilon}\right)$.

Let's now work through the machinery proposed by structuralism for this law $(L)$. We shall need a definition of a class of structures via a "set-theoretic predicate" or "Suppes predicate":

(Df1) $\quad(D, \mathbb{R}, G)$ is an stays-the-same-structure iff $G: D \rightarrow \mathbb{R}$ and, for all $d \in D, G(d)=0$.

Thus, (Df1) defines the "stays-the-same-structures". In addition, I add (Df2) and (Df3), which are definitions by comprehension, which "convert" the primitive predicate tim.inst and function symbol $\mathrm{F}$ to set constants. We compare the two approaches:

\begin{tabular}{ll} 
Propositionalist & Structuralist \\
\hline & Df1: $(D, \mathbb{R}, G)$ is an stays-the-same-structure iff $G:$ \\
& $D \rightarrow \mathbb{R}$ and, for all $d \in D, G(d)=0$. \\
& Df2: $\operatorname{Tim}=\{a \mid \operatorname{tim} . \operatorname{inst}(a)\}$. \\
& Df3: $\overline{\mathrm{F}}=\{(a, X) \in \overline{\operatorname{Tim}} \times \mathbb{R} \mid X=\overline{\mathrm{F}}(a)\}$. \\
& \\
Theoretical hypothesis (Suppes sentence Supp $(\Theta))$ \\
$\forall a(\mathrm{~F}(a) \in \mathbb{R} \wedge(\operatorname{tim} . \operatorname{inst}(a) \rightarrow \mathrm{F}(a)=0))$ & $(\overline{\operatorname{Tim}}, \mathbb{R}, \overline{\mathrm{F}})$ is a stays-the-same structure. \\
\hline
\end{tabular}

$\overline{41}$ For van Fraassen, the additional theoretical hypothesis of interest to him, for epistemological reasons, is not the claim of a theory's being true, but rather a claim of a theory's "empirical adequacy". However, I do not wish to get sidetracked into epistemology. 
The two versions are now: ${ }^{42}$

$$
\begin{array}{r}
\text { Propositionalism : } \quad \text { For all time instants } t, F(t)=0 . \\
\text { Structuralism : } \underbrace{(\overline{T i m}, \mathbb{R}, \overline{\mathrm{F}}) \text { is a stays-the-same structure }}_{\text {Suppes sentence }}
\end{array}
$$

But, as we have seen, set theory with atoms proves $(56) \leftrightarrow(57)$. They're equivalent.

So it seem hard to see why one is, in principle, preferable to the other. In practice, things might be different, and it might be easier to work with one formulation than another. However, in principle, one can go back and forth between the theory in its original "received" form, and the theoretical hypothesis (i.e., the Suppes sentence) in its "structuralist" form. For this reason, I suspect this is some sort of pseudo-debate, arguing simply about what is a "nicer way" to express provable equivalents.

\subsection{Semantic Equivalence}

Let $\sigma=\{\mathrm{P}\}$ with $\mathrm{P}:$ atom $\Rightarrow$ atom $\Rightarrow$ bool. Let $\mathrm{PO}$ be the conjunction of:

$$
\begin{gathered}
\forall a \mathrm{P}(a, a) . \\
\forall a_{1} \forall a_{2}\left(\mathrm{P}\left(a_{1}, a_{2}\right) \wedge \mathrm{P}\left(a_{2}, a_{1}\right) \rightarrow a_{1}=a_{2}\right) . \\
\forall a_{1} \forall a_{2} \forall a_{3}\left(\mathrm{P}\left(a_{1}, a_{2}\right) \wedge \mathrm{P}\left(a_{2}, a_{3}\right) \rightarrow \mathrm{P}\left(a_{1}, a_{3}\right)\right) .
\end{gathered}
$$

So, $\mathrm{PO}$ says " $\mathrm{P}$ is a partial order (on atoms)".

Then we can introduce, inside $Z_{\sigma}$, a defined satisfaction predicate Sat, some other semantic notions (e.g., value of term in an interpretation) and a gödel coding of syntactical objects as sets (say elements of $V_{\omega}$ ). In other words, we formalize the meta-theory for $L(\sigma)$ inside $\mathbf{Z A}_{\sigma}$. And then show:

Lemma 7. $\mathrm{ZA}_{\sigma} \vdash \mathrm{PO} \leftrightarrow \operatorname{Sat}((\mathbb{A}, \overline{\mathrm{P}}),\ulcorner\mathrm{PO}\urcorner)$.

Proof. The details are messy, but require only more or less standard truth-theoretic compositional moves, but all formalized inside $\mathrm{ZA}_{\sigma}:^{43}$

$$
\begin{array}{rll}
\mathrm{ZA}_{\sigma} & \vdash & \ulcorner\mathrm{P}\urcorner(\mathbb{A}, \overline{\mathrm{P}})=\overline{\mathrm{P}} \\
\mathrm{ZA}_{\sigma} & \vdash & \operatorname{Sat}((\mathbb{A}, \overline{\mathrm{P}}),\ulcorner\forall a \mathrm{P}(a, a)\urcorner) \leftrightarrow(\forall x \in \mathbb{A})\left((x, x) \in \mathrm{P}^{(\mathbb{A}, \overline{\mathrm{P}})}\right) \\
\mathrm{ZA}_{\sigma} & \vdash & \operatorname{Sat}((\mathbb{A}, \overline{\mathrm{P}}),\ulcorner\forall a \mathrm{P}(a, a)\urcorner) \leftrightarrow(\forall x \in \mathbb{A})((x, x) \in \overline{\mathrm{P}})) \\
\mathrm{ZA}_{\sigma} & \vdash & \operatorname{Sat}((\mathbb{A}, \overline{\mathrm{P}}),\ulcorner\forall a \mathrm{P}(a, a)\urcorner) \leftrightarrow(\forall x \in \mathbb{A}) \mathrm{P}(x, x) \\
\mathrm{ZA}_{\sigma} & \vdash & \operatorname{Sat}((\mathbb{A}, \overline{\mathrm{P}}),\ulcorner\forall a \mathrm{P}(a, a)\urcorner) \leftrightarrow \forall a \mathrm{P}(a, a) \\
\ldots & \cdots & \cdots \\
\mathrm{ZA}_{\sigma} & \vdash & \operatorname{Sat}((\mathbb{A}, \overline{\mathrm{P}}), x \dot{\wedge}) \leftrightarrow \operatorname{Sat}((\mathbb{A}, \overline{\mathrm{P}}), x) \wedge \operatorname{Sat}((\mathbb{A}, \overline{\mathrm{P}}), y) \\
\ldots & \cdots & \ldots \\
\mathrm{ZA}_{\sigma} & \vdash & \operatorname{Sat}((\mathbb{A}, \overline{\mathrm{P}}),\ulcorner\mathrm{PO}\urcorner) \leftrightarrow \mathrm{PO}
\end{array}
$$

42 I treat the codomain axiom for $\mathrm{F}$ as implicit, mainly for ease of presentation.

43 I am using certain kinds of "disquotation" and "compositionality". See Halbach (2014) or Cieśliński (2017) for a detailed examination of such things. 
Looking at the structure of this result:

$$
\mathrm{ZA}_{\sigma} \vdash \overbrace{\mathrm{PO}(\mathrm{P})}^{\text {Partial order axioms }} \leftrightarrow \overbrace{\operatorname{Sat}((\mathbb{A}, \overline{\mathrm{P}}),\ulcorner\mathrm{PO}\urcorner)}^{\text {System of atoms satisfies partial order axioms }}
$$

It is not difficult to see it will generalize in certain ways. In particular, with $\sigma$ purely atomic, a finitely axiomatized theory $\Theta$ in $L(\sigma)$ can be converted to a mathematically equivalent (in $\mathrm{ZA}_{\sigma}$ ) sentence $\Theta^{\text {abs }}$ (in $L\left(\sigma_{\epsilon}\right)$ ), about the "corresponding system"asserting that the system satisfies the theory $\Theta:^{44}$

SEMANTIC EQUiVALENCE

$$
\mathrm{ZA}_{\sigma} \vdash \overbrace{\Theta}^{\text {Theory }} \leftrightarrow \overbrace{\operatorname{Sat}(P,\ulcorner\Theta\urcorner)}^{\text {System satisfies theory }}
$$

\subsection{Categorical Equivalence: The Königsberg Bridge System}

Consider Example 2 again. This particular example is interesting for a number of reasons. The first has already been mentioned: we defined above the physical bridge system $P_{K}$, and noted that it an impure mathematical object: indeed, $P_{K}$ is a multigraph.

Next, I formalize this entirely in our notation and explain how this example illustrates the idea of "abstract counterparts" and what I call categorical equivalence. Fix a purely atomic application signature $\sigma=\{$ land-area, bridge, connects $\}$, with type declarations:

$$
\begin{gathered}
\text { land-area : atom } \Rightarrow \text { bool } \\
\text { bridge : atom } \Rightarrow \text { bool } \\
\text { connects : atom } \Rightarrow \text { atom } \Rightarrow \text { atom } \Rightarrow \text { bool }
\end{gathered}
$$

By comprehension and pairing, we define inside $\mathrm{ZA}_{\sigma}$ the bridge system $P_{K}$ :

$$
\begin{gathered}
\overline{\text { land-area }}:=\{a \mid \text { land-area }(a)\} . \\
\overline{\text { bridge }}:=\{a \mid \text { bridge }(a)\} . \\
\overline{\text { connects }}:=\left\{\left(a_{1}, a_{2}, a_{3}\right) \mid \text { connects }\left(a_{1}, a_{2}, a_{3}\right)\right\} \\
\mathrm{F}_{\text {connects }}:=\left\{\left(a_{1},\left\{a_{2}, a_{3}\right\}\right) \mid\left(a_{1}, a_{2}, a_{3}\right) \in \overline{\text { connects }}\right\} \\
P_{K}:=\left(\overline{\text { land-area }}, \overline{\text { bridge }}, \mathrm{F}_{\text {connects }}\right) .
\end{gathered}
$$

Define inside $\mathrm{ZA}_{\sigma}$ the (pure) mathematical object $G_{K}$ as follows:

$$
\begin{gathered}
V:=\{1,2,3,4\} \quad E:=\{5,6,7,8,9,10,11\} \\
f:=\{(5,\{1,2\}),(6,\{1,2\}),(7,\{1,3\}),(8,\{1,3\}),(9,\{1,4\}),(10,\{2,4\}),(11,\{3,4\})\} \\
G_{K}:=(V, E, f)
\end{gathered}
$$

\footnotetext{
44 The reader might note the analogy with disquotation sentences for a truth predicate: $\mathrm{T}(\ulcorner\phi\urcorner) \leftrightarrow \phi$. This connection is not a coincidence! We could simply define $\mathrm{T}(X)$ to mean: Sat $((\mathbb{A}, \overline{\mathrm{P}}), X)$. And then: $\mathrm{ZA}_{\sigma} \vdash \mathrm{T}(\ulcorner\phi\urcorner) \leftrightarrow \phi$, for all $\phi \in L(\sigma)$.
} 
Then $G_{K}$ is a multigraph with vertices $V$, edges $E$ and connection function $f:^{45}$

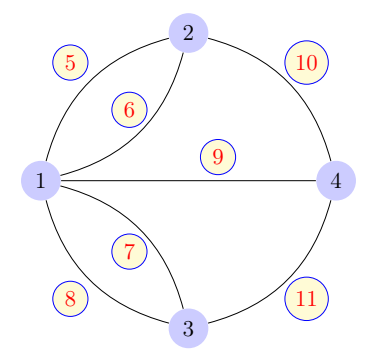

We need a sentence in $L(\sigma)$ which describes the bridge-connection relationships amongst the four land-areas in Königsberg.

Definition 18 (The Königsberg sentence). First, define the following eight $L(\sigma)$ formulas, $\theta_{i}: 46$

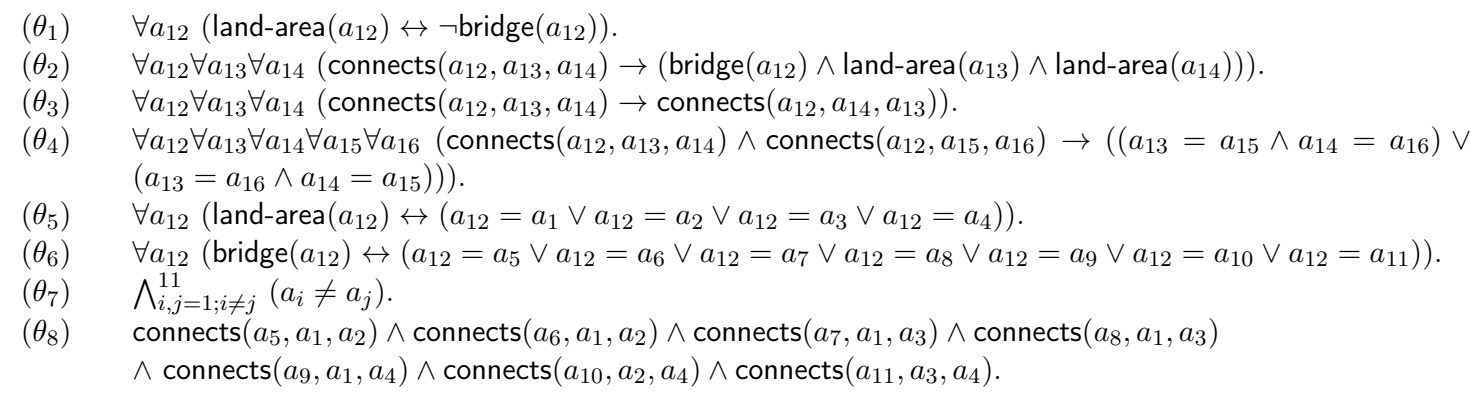

The Königsberg sentence $\phi_{K}$ is defined as follows, by taking the conjunction of the $\theta_{i}$, and then existentially quantifying the eleven free variables:

$$
\phi_{K}:=\exists a_{1} \ldots \exists a_{11} \bigwedge_{i=1}^{8} \theta_{i}
$$

Lemma 8. $G_{K}$ is a model of $\phi_{K}$ and any model of $\phi_{K}$ is isomorphic to $G_{K}$.

Proof. One first shows that $G_{K}$ is a model of $\phi_{K}$ directly. Next, assuming $M \models \phi_{K}$, one can show, by looking at what each axiom requires, that $M$ must be isomorphic to $G_{K}$.

45 I.e., $f(5)=\{1,2\}$, etc. That is, edge labelled 5 connects vertices labelled 1 and 2 . Etc.

46 These axioms (if we treat the free variables $a_{1}, \ldots, a_{11}$ as constants) are true of $P_{K}$. Moreover, they provide a categorical description of the structured system $P_{K}$ we are interested in. I call the first four axioms "structural axioms": any (undirected) multigraph satisfies these. Axiom $\left(\theta_{5}\right)$ is the vertex axiom: the vertices - i.e., land-areas - are $a_{1}, \ldots, a_{4}$. Axioms $\left(\theta_{6}\right)$ is the edge axioms: the edges-i.e., bridges - are $a_{5}, \ldots, a_{11}$. Axiom $\left(\theta_{7}\right)$ states that these are all distinct. Axiom $\left(\theta_{8}\right)$ is the "connection axiom" stating how the bridges and land-areas are connected in this specific multigraph. 
So $\phi_{K}$ itself is a categorical description of the Königsberg bridge system. Following our discussion of the semantic equivalence above, we have:

Lemma 9. $\mathrm{ZA}_{\sigma} \vdash \phi_{K} \leftrightarrow \operatorname{Sat}\left(P_{K},\left\ulcorner\phi_{K}\right\urcorner\right)$.

But Lemma 8 implies:

Lemma 10. $P_{K}$ is a model of $\phi_{K}$ iff $P_{K}$ is isomorphic to $G_{K}$.

Lemma 10 can be formalized inside $\mathbf{Z A}_{\sigma}$ :

Lemma 11. $\mathrm{ZA}_{\sigma} \vdash \operatorname{Sat}\left(P_{K},\left\ulcorner\phi_{K}\right\urcorner\right) \leftrightarrow\left(P_{K} \cong G_{K}\right)$.

Hence, by Lemmas 9 and 11 we conclude:

Lemma 12. $\mathrm{ZA}_{\sigma} \vdash \overbrace{\phi_{K}}^{\text {Sentence }} \leftrightarrow \overbrace{\left(P_{K} \cong G_{K}\right)}^{\text {Abstract equivalent }}$.

In summary, we have two (contingent) sentences which are equivalent modulo set theory (with atoms):

$\phi_{K}$ (in $\left.L(\sigma)\right)$ says: $\quad$ "There are seven bridges, four land-areas and certain connection relations holding between them".

$P_{K} \cong G_{K}\left(\right.$ in $\left.L\left(\sigma_{\epsilon}\right)\right)$ says: "The Königsberg bridge system $P_{K}$ is isomorphic to $G_{K}$ ".

In this particular case, we have what I call categorical equivalence:

$$
\mathrm{ZA}_{\sigma} \vdash \overbrace{\phi_{K}}^{\text {Königsberg sentence }} \leftrightarrow \overbrace{\left(P_{K} \cong G_{K}\right)}^{\text {Rep. Hypothesis }}
$$

On the right-hand side, we have an isomorphism claim which I call a Representation Hypothesis. The more general phenomenon is the following. For finitely axiomatized categorical theories $\Theta$ in a purely atomic signature $\sigma$ whose categoricity is provable in set theory, we obtain: ${ }^{47}$

Categorical equivalence

$$
\text { ZFCA }_{\sigma} \vdash \overbrace{\Theta}^{\text {Theory }} \leftrightarrow \overbrace{(P \cong M)}^{\text {Repres. Hypothesis }}
$$

\subsection{Example of Categorical Equivalence: Second-Order Geometry}

David Hilbert (Hilbert (1899)) formulated Euclidean geometry as a second-order theory and proved a representation theorem to the effect that any (full) model of the theory is isomorphic to $\mathbb{R}^{3}$, with certain additional geometric relations defined on $\mathbb{R}^{3}$. Alfred

\footnotetext{
47 This leaves open the possibility that $\Theta$ may be categorical, but not provably so. Sentences of the form " $\Theta$ is categorical" (including when $\Theta$ is "second-order": i.e., has set quantifiers) are hardly elementary, or even arithmetic sentences, so this must be a real possibility.
} 
Tarski and his school simplified the axioms, resulting in systems I call $\mathrm{Geom}_{n}^{d}$, for $d$ dimensional geometry, with $n=1$ or $n=2$. $\mathrm{Geom}_{1}^{d}$ is a first-order system and $\mathrm{Geom}_{2}^{d}$ is second-order. The axioms for Geom ${ }_{1}^{d}$ are given in Tarski (1959) (pp. 19-20). The axioms for $\mathrm{Geom}_{2}^{d}$ are obtained by changing the elementary continuity axioms (Tarski (1959), p. 20) into a corresponding single axiom, using a set quantifier over points. Tarski proved a representation theorem for the first-order theories (Tarski (1959): Theorem 1, pp. 2122). Focusing on the second-order version, the Hilbert-Tarski representation theorem says: ${ }^{48}$

Theorem 2. $M$ is a full model of $\mathrm{Geom}_{2}^{d}$ iff $M$ is isomorphic to $\left(\mathbb{R}^{d}, B_{\mathbb{R}^{d}}, C_{\mathbb{R}^{d}}\right)$.

It is intuitively clear that this is provable inside set theory. To put this into our formalization, fix an application signature $\sigma=\{\mathrm{B}, \mathrm{C}\}$ with type declarations: $\mathrm{B}$ : atom $\Rightarrow$ atom $\Rightarrow$ atom $\Rightarrow$ bool and $C:$ atom $\Rightarrow$ atom $\Rightarrow$ atom $\Rightarrow$ atom $\Rightarrow$ bool. Let $\mathrm{Geom}_{2}^{d=3}(B, C)$ be 2 nd order Hilbert-Tarski geometry with $d=3$ using these primitives.

Then we have a semantic equivalence (76) and the Hilbert-Tarski Representation Theorem (77) as theorems of set theory with atoms: ${ }^{49}$

$$
\begin{gathered}
\mathrm{ZA}_{\sigma} \vdash \operatorname{Geom}_{2}^{d=3}(\mathrm{~B}, \mathrm{C}) \leftrightarrow \operatorname{Sat}_{2}\left((\mathbb{A}, \overline{\mathrm{B}}, \overline{\mathrm{C}}),\left\ulcorner\operatorname{Geom}_{2}^{d=3}(\mathrm{~B}, \mathrm{C})\right\urcorner\right) \\
\mathrm{ZA}_{\sigma} \vdash \operatorname{Sat}_{2}\left(M,\left\ulcorner\operatorname{Geom}_{2}^{d=3}(\mathrm{~B}, \mathrm{C})\right\urcorner\right) \leftrightarrow\left(\exists \varphi: \mathbb{A} \rightarrow \mathbb{R}^{3}\right)\left(M \stackrel{\varphi}{\cong}\left(\mathbb{R}^{3}, B_{\mathbb{R}^{3}}, C_{\mathbb{R}^{3}}\right)\right)
\end{gathered}
$$

Together these imply another categorical equivalence: ${ }^{50}$

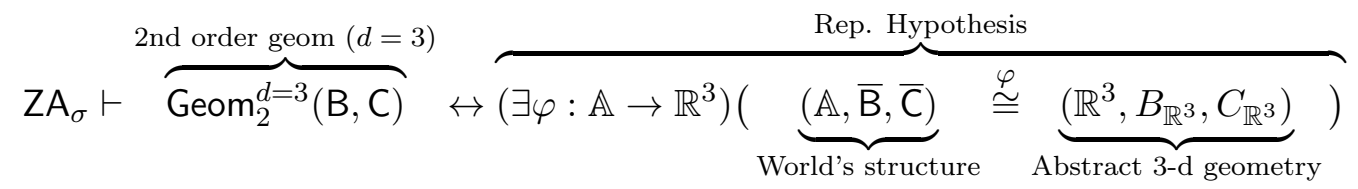

We may, as Albert Einstein did (see Einstein (1921)), see the geometric theory $\mathrm{Geom}_{2}^{d=3}(\mathrm{~B}, \mathrm{C})$ as an interpreted physical theory: it says the points of real physical space are betweenness-related, and congruence-related, thus-and-so. ${ }^{51}$ Yet realizing of course the heavily mathematicized nature of contemporary geometry within pure mathematics itself, Einstein raises the issue of applicability in Einstein (1921):

$\overline{48} B_{\mathbb{R}^{d}}$ and $C_{\mathbb{R}^{d}}$ are betweenness and congruence relations defined on $\mathbb{R}^{d}$. See Tarski (1959), p. 21, for their definitions (as applied to an arbitrary real-closed field $F$. Here that field is $\mathbb{R}$ ). I have changed notation a little bit.

49 The subscript on the (model-theoretic) satisfaction predicate $\operatorname{Sat}_{2}$ is there to indicate that $\operatorname{Sat}_{2}(M, \ldots)$ means " $M$ is a full model of ...".

50 One can repeat this trick for any finitely axiomatized categorical second-order theory whose categoricity is provable in set theory. For example, $\mathrm{PA}_{2}$ and $\mathrm{RCF}_{2}$.

51 A claim which ultimately has empirical content. The surface area $A$ of any sphere with radius $R$ metres around some point $p$ (imagine a gigantic spherical "balloon", millions of kilometres in radius, centred on the Sun) should satisfy $A=4 \pi R^{2}$ (in metres $^{2}$ ). If $A<4 \pi R^{2}$ or $A>4 \pi R^{2}$, then the (spatial) geometry is non-Euclidean. 
At this point an enigma presents itself which in all ages has agitated inquiring minds. How can it be that mathematics, being after all a product of human thought which is independent of experience, is so admirably appropriate to the objects of reality? (Einstein (1921): 28)

Einstein goes on to describe the then-contemporary (Hilbertian) views, as "advocated by modern axiomatics":

This view of axioms, advocated by modern axiomatics, purges mathematics of all extraneous elements, and thus dispels the mystic obscurity which formerly surrounded the principles of mathematics. But a presentation of its principles thus clarified makes it also evident that mathematics as such cannot predicate anything about perceptual objects or real objects. In axiomatic geometry the words "point", "straight line", etc., stand only for empty conceptual schemata. That which gives them substance is not relevant to mathematics. (Einstein (1921): 30-31)

Einstein notes the puzzle concerning how this more structural view of geometry, which had become prevalent under the influence of Hilbert (1899) and others was reconnected back up to the physics. ${ }^{52}$

The solution that Einstein gives is quoted below, at the beginning of Section 8: we require an additional claim involving "the co-ordination of real objects of experience with the empty conceptual frame-work of axiomatic geometry". This is what I call a Representation Hypothesis below. The explanation of how the two ways of putting things are "connected" is given by our (78): the physical theory $\operatorname{Geom}_{2}^{d=3}(\mathrm{~B}, \mathrm{C})$ may be reformulated equivalently, in a "more structural way", as a Representation Hypothesis, saying that there is an isomorphic correspondence between these points equipped with their physical structure, viz. $(\mathbb{A}, \bar{B}, \bar{C})$, and the abstract system $\left(\mathbb{R}^{3}, B_{\mathbb{R}^{3}}, C_{\mathbb{R}^{3}}\right)$. It is intimately related also to what the logical empiricists began to call "correspondence rules" or "bridge principles".

\section{Application Conditionals}

I find it quite amazing that it is possible to predict what will happen by mathematics, which is simply following rules which really have nothing to do with the original thing. (Feynman (1965): 171.)

\subsection{Definition}

Definition 19. Let $\phi_{1}$ and $\phi_{2}$ be $L\left(\sigma_{\epsilon}\right)$-formulas (which may have free variables). If

$$
\mathrm{ZFCA}_{\sigma} \vdash \phi_{1} \rightarrow \phi_{2}
$$

we say that $\phi_{1} \rightarrow \phi_{2}$ is an application conditional (provable in ZFCA ). .

\footnotetext{
52 This was a topic of enormous interest to, and huge stimulus to, the newly forming group of logical empiricists, Moritz Schlick, Rudolf Carnap and Hans Reichenbach. See Schlick (1918), Carnap (1922), Reichenbach (1924).
} 
This looks very trivial. And indeed is, in some cases. For example, $\forall x(x=x) \rightarrow$ $\forall x(x=x)$ is an application conditional, as is any logical validity of the form $\phi_{1} \rightarrow \phi_{2}$. And if $\phi_{2}$ is a theorem of ZFCA $\sigma$, then, again trivially, so is $\phi_{1} \rightarrow \phi_{2}$.

However, it becomes non-trivial when $\phi_{1}$ and $\phi_{2}$ are not theorems of ZFCA $\sigma$. And this is the standard situation in applied mathematics: in that setting, application conditionals turn up everywhere. The reason is the following. In applied mathematics, we frequently mathematically reason from some assumptions about, e.g., some physical function $F$ (i.e., a mixed function) and/or some "physical" set $X$ and/or relation $R$ (including impure sets and relations of concrete things) to conclusions about that function $F$, and/or set $X$ and/or relation $R .^{53}$ Suppose we schematically represent the premise as $\phi_{1}(F, \ldots)$ and the conclusion as $\phi_{2}(F, \ldots)$. In general, $\phi_{1}(F, \ldots)$ and $\phi_{2}(F, \ldots)$ are not theorems of applied mathematics. However, we may reason mathematically from $\phi_{1}(F, \ldots)$ to $\phi_{2}(F, \ldots)$ within applied mathematics.

To put it as a metaclaim about provability in the base theory:

$$
\mathrm{ZFCA}_{\sigma} \vdash \phi_{1}(F, \ldots) \rightarrow \phi_{2}(F, \ldots)
$$

In some cases, the $F, \ldots$ in premise $\phi_{1}(F, \ldots)$ and conclusion $\phi_{2}(F, \ldots)$ are free variables. In some cases, $F, \ldots$ may be function symbols, predicates or constants in the application signature. Either way, it is not usually the case that these premises and conclusions are provable in $\mathrm{ZFCA}_{\sigma}$. And it is not usually the case that these sentences $\phi_{1}, \phi_{2}$ are themselves mathematics-free.

\subsection{Examples: Differential equations}

Examples in mathematical physics abound. Many have the following form:

$$
\mathrm{ZFCA}_{\sigma} \vdash[\overbrace{\operatorname{Law}(F, \ldots)}^{\text {law/differential equation }} \wedge \overbrace{\mathrm{BC}(F, \ldots)}^{\text {boundary condition }}] \rightarrow \overbrace{\operatorname{Sol}(F, \ldots)}^{\text {solution }} .
$$

Suppose we have a simple first-order differential equation and along with a boundary condition, such as: ${ }^{54}$

$$
\begin{gathered}
\partial_{t} F=k F(t) \\
F(0)=p
\end{gathered}
$$

Then it is standard exercise to deduce the form of its solutions:

$$
F(t)=p e^{k t}
$$

\footnotetext{
53 The function $F$ might be a temperature function, or an electric field, or a wavefunction, or ...; the set $X$ might be the set of impacts between a billiard ball and the cushion, or the set of voters in a region, or ....

54 Here, we have some otherwise unspecified function $F: \mathbb{R} \rightarrow \mathbb{R}$, and $k \in \mathbb{R}$ is some fixed parameter.
} 
In such cases, clearly the premises and conclusion are not theorems of applicable mathematics. However, the whole conditional is such a theorem: ${ }^{55}$

$$
\mathrm{ZFCA}_{\sigma} \vdash[\cdots \wedge \overbrace{(\forall t \in \mathbb{R})\left(\partial_{t} F(t)=k F(t)\right)}^{\text {differential equation }} \wedge \overbrace{F(0)=p}^{\text {boundary condition }}] \rightarrow \overbrace{\forall t \in \mathbb{R}\left(F(t)=p e^{k t}\right)}^{\text {solution }}) .
$$

Example 4. Schrödinger's Equation and the Quantized Energy Spectrum

I return to the example mentioned at the end of Subsection 1.4. First, the timeindependent Schrödinger Equation, specialized to the hydrogen atom, is, roughly: ${ }^{56}$

$$
(\forall x: \text { Space })\left(\left(-\frac{\hbar^{2}}{2 m_{e}} \nabla^{2}-\frac{e^{2}}{4 \pi \epsilon_{0}|x|}\right) \Psi(x)=E \Psi(x)\right)
$$

Clearly, applied mathematics does not prove the (physical) Schrödinger equation (85), which, after all, is contingent. But applied mathematics does prove the conditionalthat if the Schrödinger equation holds for a (possibly mixed) function $F$ on some set or structure $X$ (which may or may not be spacetime), satisfying certain conditions, then the solutions are thus-and-so, or certain properties of such solutions hold:

$$
[F: X \rightarrow \mathbb{C} \wedge \cdots \wedge \overbrace{(\forall x: X)\left(\left(-a \nabla^{2}-\frac{b}{|x|}\right) F(x)=E F(x)\right)}^{\text {Time Indep Schrödinger equation }} \wedge E<0] \rightarrow \overbrace{\left(\exists n \in \mathbb{N}_{>0}\right)\left(E=-\frac{c}{n^{2}}\right)}^{\text {Quantized spectrum }}
$$

An application conditional like (86) may require a substantial level of mathematical sophistication to prove. Indeed, textbooks and monographs in mathematical physics probably devote over $90 \%$ of their material to developing such techniques.

It is now easy to see how the application conditional (86) is then applied to the physical law (85) itself. It is merely an example of quantifier instantiation, followed by modus ponens; doing this (and plugging in the correct value for $c$, given the specified parameters) we obtain:

$$
E<0 \rightarrow\left(\exists n \in \mathbb{N}_{>0}\right)\left(E=-\frac{m_{e} e^{4}}{2\left(4 \pi \epsilon_{0}\right)^{2} \hbar^{2}} \frac{1}{n^{2}}\right)
$$

This says the bound states $(E<0)$ have precisely these energy eigenvalues.

55 The dots "..." in the antecedent stand for a a couple of implicit structural conditions " $F$ is a function on the reals $(F: \mathbb{R} \rightarrow \mathbb{R})$ and $F$ is differentiable".

56 Here I simplify a fair amount and use some standard physics notation. The " $|x|$ " means the length of the segment from the point $x$ to some implicit origin $O$, centred in the proton. Normally, this equation is written in terms of the co-ordinate representation of $\Psi$ in polar co-ordinates - say $\psi(r, \theta, \phi)$ - with $r=0$ being the proton's location, and then $|x|$ is replaced by $r$, but I wish to stress that $\Psi$ really is a (mixed) function on space, even though its co-ordinate representation is a (pure) function $\mathbb{R}^{3} \rightarrow \mathbb{C}$. 


\subsection{Remark on the "Mapping Accounts"}

Recent advocates of "mapping account(s)" of applied mathematics have argued that, whenever mathematics is applied, this always must be reducible to there being a "representational mapping", say a morphism

$$
h: M_{\mathrm{emp}} \rightarrow M
$$

from an "empirical setup" (or "assumed structure") $M_{\mathrm{emp}}$ to a pure mathematical structure $M .{ }^{57}$ My focus here is on the view set out in Bueno \& Colyvan (2011) and Bueno \& French (2018).

In the case of geometry (e.g., Subsection 6.5), or in the case of the Königsberg bridge problem (e.g., Example 6.4, Subsection 6.4, Example 5), one can see that something like this is indeed the case. ${ }^{58}$

However, in examples like the above - and differential equations in general in mathematical physics, involving mixed quantity functions - I don't see anything like such a "representational mapping". What, for example, is $M_{\mathrm{emp}}$ ? What is $h$ ? The wavefunction? How is the wavefunction a representational mapping? What relations does it preserve or represent?

Instead, it seems we have (i), an application conditional (86), a theorem of applied mathematics (i.e., provable in a system like $\mathrm{ZFCA}_{\sigma}$ ), and (ii), a law of physics (85). The conditional is universally quantified with what are, in effect, set and function quantifiers. I am then able to instantiate the initial quantifiers of this theorem on the set terms from the physical law itself (85) - i.e., set terms which denote the (mixed) wavefunction and the (impure) set of space points. Consequently, I may infer the required conclusion, (87), regarding the energy spectrum of hydrogen.

In fact, I would estimate a gigantic proportion of applied mathematics fits this very mould: universally quantified application conditionals (provable in the applied mathematics base theory), combined with specific (contingent) laws containing set or function constants (denoting certain mixed physical quantities, often measurable ones) which can be used to instantiate the relevant conditional.

It is not clear to me how this fits into the "mapping account". Perhaps it is being implicitly assumed that the physical wavefunction $\Psi$ (of the electron) is itself a "representational mapping"? But how is that so? This particular claim does happen to be a central component of Field's Programme, and Field aims to establish such results in Field

57 See Pincock (2007), Pincock (2012), Bueno \& Colyvan (2011) and Bueno \& French (2018). The Königsberg Problem is raised and discussed in some detail in both Pincock (2007) and Pincock (2012).

58 In both cases, I also defined the "assumed structure". For geometry, the assumed structure is $(\mathbb{A}, \overline{\mathrm{B}}, \overline{\mathrm{C}})$. For the Königsberg Problem, the assumed structure is $P_{K}$, the Königsberg multigraph. I should mention also that the Königberg problem can be solved without invoking any mapping or isomorphism. One may simply reason directly about $P_{K}$ and infer from its properties that each vertex has odd degree, and thus that it lacks a Euler cycle, without a detour involving an abstract copy (i.e., $G_{K}$ ) of $P_{K}$. In fact, the problem can be solved without mathematics at all, but that brings in considerations of conservativeness. 
(1980), by showing that physical fields can be "nominalized". So the only way I can see the "mapping account" as being in accord with the widespread formulation of physical laws as differential equations is that it presupposes the success of Field's Programme.

\subsection{Example of Mathematical Explanation}

Our Schrödinger Equation example also seems to provide an example of mathematical explanation: Why are the energy levels of the hydrogen atom quantized? The answer is: first, the orbital wavefunctions of the hydrogen atom are eigenstates satisfying the time-independent Schrödinger equation; second, the negative eigenvalue spectrum (for these eigenstates) is quantized. The first fact is contingent (and only holds to a certain approximation). But the second fact, i.e., (86), is a theorem of the base theory for applied mathematics.

\section{Representation and Transfer}

To be able to make such assertions, geometry must be stripped of its merely logical-formal character by the co-ordination of real objects of experience with the empty conceptual frame-work of axiomatic geometry. To accomplish this, we need only add the proposition: -

Solid bodies are related, with respect to their possible dispositions, as are bodies in Euclidean geometry of three dimensions.

Then the propositions of Euclid contain affirmations as to the relations of practically-rigid bodies.

Geometry thus completed is evidently a natural science; we may in fact regard it as the most ancient branch of physics. (Einstein (1921): 31-32. Emphasis added)

\subsection{Isomorphism and Transfer}

A standard result in model theory, the Isomorphism Theorem, is the following:

Theorem 3. Let $\sigma$ be a signature and let $A, B$ be $\sigma$-structures. Then

$$
\text { If } A \stackrel{F}{\cong} B \text { then, for any } \phi \in L(\sigma), A \models \phi \text { iff } B \models \phi .
$$

There is a sense in which " $A \models \phi$ " expresses that the structure $A$ has a certain "structural property" (viz: the structural property defined by $\phi$ ). ${ }^{59}$ So, the Isomorphism Theorem states that if $A \cong B$, then $A$ and $B$ have the same structural properties. One can express this a little more intuitively by talking of transfer:

\footnotetext{
59 A "structural property" of $A=\left(D, R_{1}, \ldots\right)$ is one that doesn't depend on aspects of the intrinsic nature of the elements of $\operatorname{dom}(A)$, but only on the properties and relations $R_{i}$ explicitly specified in the structure $A$. See Korbmacher \& Schiemer (2018) for a discussion of such properties and an analysis of them in terms of definability in infinitary languages.
} 
If $A \cong B$, any structural property of $A$ transfers to a structural property of $B$ (and vice versa).

This yields another, extremely common, phenomenon in applied mathematics. We define, usually by comprehension, an impure physical system $P$. We then state a contingent representation hypothesis: ${ }^{60}$

$$
\text { Representation Hypothesis: } \exists F(P \stackrel{F}{\cong} M) \text {. }
$$

From this hypothesis, we may deduce (within applied mathematics):

Transfer Principle: If $M$ satisfies condition $\phi, P$ satisfies condition $\phi$.

Schematically:

$$
\text { Transfer Principle: } \phi(M) \rightarrow \phi(P) .
$$

We have the physical system $P$ (the physical system is an impure mathematical object, sometimes involving mixed functions too). This physical system is represented by an abstract mathematical object $M$ via an isomorphism. Since we may already have some large library of results about that object $M$ (e.g., because it belongs to a well-known class of objects satisfying similar conditions), we can "transfer" such results back to $P .{ }^{61}$

\subsection{Examples}

\section{Example 5. Königsberg Bridges}

I return again to Example 2 and Section 6.4. There, we fixed a purely atomic application signature $\sigma=\{$ bridge, land-area, connects $\}$; we defined the Königsberg bridge system $P_{K}$; we expressed the "relevant empirical facts" in the Königsberg sentence $\phi_{K}$; we defined an abstract multigraph $G_{K}$; we proved that $P_{K}$ is a model of $\phi_{K}$ and that $\phi_{K}$ is categorical; and we sketched the result that this in formalizable inside $Z_{\sigma}$ :

$$
\mathrm{ZA}_{\sigma} \vdash \phi_{K} \leftrightarrow\left(P_{K} \cong G_{K}\right)
$$

Here, the mathematical sentence $\left(P_{K} \cong G_{K}\right)$, which is an abstract equivalent of $\phi_{K}$, is a Representation Hypothesis: therefore, we obtain transfer principles from it. In particular, the following property of the (pure) multigraph $G_{K}$, provable in $\mathrm{ZA}_{\sigma}$ (in $\mathrm{ZA}_{\sigma}^{-\infty}$ in fact):

Lemma 13. $G_{K}$ has no Euler cycle.

60 Here the representation is an isomorphism. But it is clear that sometimes weaker morphisms, homomorphisms and embeddings, are in play in applied mathematics.

61 In this case, structures $A$ and $B$ are isomorphic. What if there is merely a homomorphism $f: A \rightarrow B$ ? Or an embedding, say? Then the class of transferable properties is smaller. The pattern for groups is analysed in Lyndon (1959). For example if $f: G_{1} \rightarrow G_{2}$ is a surjective homomorphism of groups, then $G_{2}$ is Abelian if $G_{1}$ is; and $G_{2}$ is cyclic if $G_{1}$ is; etc. 
Proof. $G_{K}$ is a connected multigraph with 4 vertices and each vertex has odd degree. By Theorem 1, $G_{K}$ has no Euler cycle.

We may therefore combine this with the above, and transfer this property to the physical bridge system $P_{K}$ :

Lemma 14. $\mathrm{ZA}_{\sigma} \vdash\left[\phi_{K} \rightarrow\left(P_{K}\right.\right.$ has no Euler cycle $\left.)\right]$.

We have a proof in applied mathematics of the conditional claim that: if $\phi_{K}$ is true, then $P_{K}$ has no Euler cycle. Since $\phi_{K}$ is (empirically) true, we conclude that $P_{K}$ has no Euler cycle. ${ }^{62}$

I must stress that applied mathematics $\mathrm{ZA}_{\sigma}$ does not, of course, prove the contingent sentence " $P_{K}$ has no Euler cycle" (about an impure structure). It proves the conditional: "If some vertex in $P_{K}$ has odd degree, then $P_{K}$ has no Euler cycle". Indeed, this-i.e., its provability in applied mathematics - is what licences any counterfactual conclusions we might draw about the bridges at present, or in future.

Example 6. Geometry

I return to Subsection 6.5, concerning second-order geometry and categorical equivalence. Inside our applied mathematics base theory - set theory with atoms - we can prove the abstract equivalence:

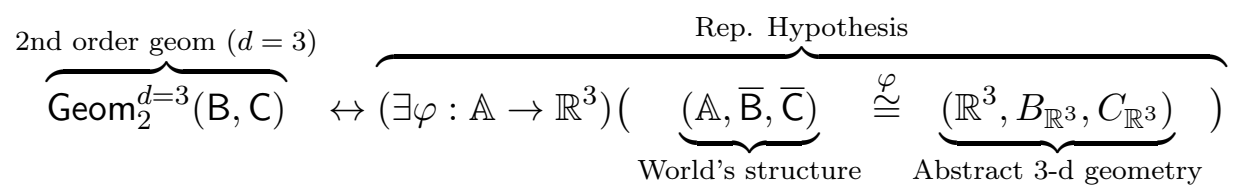

Thus if I think of my overall scientific theory $T$ of the geometry of space as follows: ${ }^{63}$

$$
T:=\mathrm{ZFCA}_{\sigma}+\mathrm{Geom}_{2}^{d=3}(\mathrm{~B}, \mathrm{C}),
$$

I can therefore prove in $T$ the Representation Hypothesis:

$$
T \vdash\left(\exists \varphi: \mathbb{A} \rightarrow \mathbb{R}^{3}\right)(\underbrace{(\mathbb{A}, \overline{\mathrm{B}}, \overline{\mathrm{C}})}_{\text {World's structure }} \stackrel{\varphi}{\cong} \underbrace{\left(\mathbb{R}^{3}, B_{\mathbb{R}^{3}}, C_{\mathbb{R}^{3}}\right)}_{\text {Abstract 3-d geometry }})
$$

So, if I happen to know some (pure, and structural) mathematical fact about $\left(\mathbb{R}^{3}, B_{\mathbb{R}^{3}}, C_{\mathbb{R}^{3}}\right)$, I can transfer it back to the (impure) physical system $(\mathbb{A}, \overline{\mathrm{B}}, \overline{\mathrm{C}})$. To take a standard example that arises in physics lectures, we know from the linear algebra of vector spaces that there are not four vectors $\mathbf{v}_{1}, \mathbf{v}_{2}, \mathbf{v}_{3}, \mathbf{v}_{4}$ in $\mathbb{R}^{3}$ which are mutually at right angles. For if I have three $\mathbf{v}_{1}, \mathbf{v}_{2}, \mathbf{v}_{3}$ meeting this condition, then they form a basis (for

62 Although $\phi_{K}$ was true, it is, apparently, no longer true; some of the bridges of Königsberg at the time of Euler have been knocked down.

${ }^{63}$ We know, course, from Einstein, that $T$ is not true. The flat spatial geometry $\operatorname{Geom}_{2}^{d=3}(\mathrm{~B}, \mathrm{C})$ is in some sense "approximately true" for smallish regions of space around a point. 
the tangent space), and therefore any extra vector $\mathbf{w}$ that I happen to consider is linearly dependent on them somehow (there are reals $\alpha_{1}, \alpha_{2}, \alpha_{3}$ st: $\mathbf{w}=\alpha_{1} \mathbf{v}_{1}+\alpha_{2} \mathbf{v}_{2}+\alpha_{3} \mathbf{v}_{3}$ ).

By transfer, this means that there cannot be four line segments (say all rooted at some point $O)$ in $(\mathbb{A}, \overline{\mathrm{B}}, \overline{\mathrm{C}})$-i.e., in physical space - all mutually at right angles. But there can be three, given by one's thumb, forefinger and second finger:

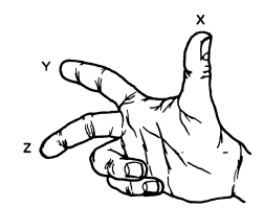

But no matter how one tries to point one's third finger, it cannot point perpendicularly to the three given directions. ${ }^{64}$

\section{Summary}

A major point I want to make is that a simple standard formalization of most theories in the empirical sciences is not possible. The source of the difficulty is easy to describe. Almost all systematic scientific theories of any interest or power assume a great deal of mathematics as part of their substructure. There is no simple or elegant way to include this mathematical substructure in a standard formalization that assumes only the apparatus of elementary logic. (Suppes (1992): 207)

I have described a many-sorted base theory for applied mathematics-ZFCA : Zermelo-Fraenkel set theory (with Choice) with atoms, over an underlying sort system $S=\{$ bool, atom, set, global $\}$ - showing how it accommodates reasoning about impure mathematical objects (sets and relations of atoms; structures built from them) and mixed relations and functions. The formalism incorporates what I have called an "application signature" $\sigma$ (which can be varied from case to case). The examples given above illustrate the following five features of applied mathematics:

64 One may, of course, take this empirical observation and read it backwards: as empirical evidence for $d=3$ in our underlying geometric system $\operatorname{Geom}_{2}^{d}(\mathrm{~B}, \mathrm{C})$. This is precisely what one hears in a physics lecture. "Why is $d=3$ ? How do we know? Why isn't $d=4$ or $d=25$ ?", followed by the three finger explanation. Of course, this argument may break down if there are teeny extra dimensions, too small for us to notice them as a new direction into which to "point". The idea that (spatial) $d=4$ in fact was introduced by Theodor Kaluza in 1921 (Kaluza (1921)) and extended by Oskar Klein in 1926 (Klein (1926)), suggesting the extra dimension is curled up in a tiny circle with subatomic radius. In such "Kaluza-Klein theories", by imposing Einstein's equations on the $4+1$-metric, one obtains Maxwell's equations as a consequence! The idea that $d=25$ arises in the quantum theory of (bosonic) strings. The quantum theory of a bosonic string in $N$ dimensions only works if $N=25+1$. 
Comprehension principles Application conditionals

Representation hypotheses Transfer principles Abstract equivalents

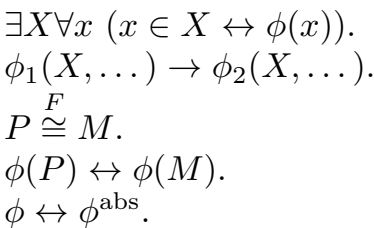
$\phi_{1}(X, \ldots) \rightarrow \phi_{2}(X, \ldots)$.

$P \stackrel{F}{\cong} M$.

$\phi \leftrightarrow \phi^{\mathrm{abs}}$.

To test the adequacy of this approach, including identifying when the features CARTA appear, one may take any law of physics, or quantitative law, or statistical reasoning, or discrete mathematics reasoning, and see if it can be formalized in $\mathrm{ZFCA}_{\sigma}$, at least in principle. One must fix a signature $\sigma$, and then formalize the relevant laws, assumptions, etc., in this signature. ${ }^{65}$ There is no requirement that the signature $\sigma$ be purely atomic (although that case is philosophically interesting, as Field (1980) made clear).

In a second paper, I intend to explain certain meta-logical features of applied mathematics - conservativeness, non-conservativeness, and speed-up. ${ }^{66}$

\section{A Four-Sorted System}

\section{A.1 Well-Formed Terms and Primitive Formulas}

Definition 20 (Well-formed). Let $\sigma$ be an application signature over $S$. We recursively define the well-formed terms and well-formed primitive formulas:

(1) Each constant (including $T, \perp$ ) and variable is a well-formed primitive term.

(2) If $\mathrm{F}$ is an $n$-ary function symbol in $\sigma$ and $t_{1}, \ldots, t_{n}$ are well-formed terms, then $\mathrm{F}\left(t_{1}, \ldots, t_{n}\right)$ is a well-formed term iff $\tau_{i}(\mathrm{~F})=\tau\left(t_{i}\right)$; and, if well-formed, the term $\mathrm{F}\left(t_{1}, \ldots, t_{n}\right)$ has sort $\tau_{n+1}(\mathrm{~F})$.

(3) If $\mathrm{P}$ is an $n$-ary predicate in $\sigma, t_{1}, \ldots, t_{n}$ are well-formed terms, then $\mathrm{P}\left(t_{1}, \ldots, t_{n}\right)$ is a well-formed primitive formula iff either $\tau_{i}(\mathrm{P})=\tau\left(t_{i}\right)$ or $\tau\left(t_{i}\right)$ is global.

Note: the additional clause in (3) implies that if $x_{1}, \ldots, x_{n}$ are global variables, then $\mathrm{P}\left(x_{1}, \ldots, x_{n}\right)$ is always a well-formed primitive formula.

\section{A.2 Definition of the language $L\left(\sigma_{\epsilon}\right)$}

Definition 21 (Definition of $L\left(\sigma_{\epsilon}\right)$ ). In stages: (1) Variables: Each sort has its own stock of variables $\left(\mathbf{b}_{i}, a_{i}, X_{i}, x_{i}\right)$. (2) Identity 83 Membership Predicates: There is a global identity predicate $=$ and a global membership predicate $\in$, each with the type declaration: global $\Rightarrow$ global $\Rightarrow$ bool. (3) Terms: primitive terms are defined to be variables and

${ }_{65}$ One could extend the sort system $S$, if one likes, to make it more "user-friendly". E.g., in geometry, sorts point and region treated as atoms; in pure mathematics, sorts for $\mathbb{N}, \mathbb{Z}, \mathbb{Q}, \mathbb{R}$, and $\mathbb{C}$, treated as atoms. Or one might include sorts for special kinds of sets, relations, functions, or structures.

66 I am grateful to two referees who provided very helpful comments and suggestions. I am grateful to Ali Enayat and Christopher Menzel for suggestions. This work was supported by a grant from the National Science Centre (NCN) in Kraków (2018/29/B/HS1/01832). 
constants; terms are defined to be primitive terms, along with, for terms $t_{1}, \ldots, t_{n}$ and $n$ ary function symbol $\mathrm{F}$, the function terms $\mathrm{F}\left(t_{1}, \ldots, t_{n}\right)$ constructed inductively, matching the sort restrictions given above. ${ }^{67}$ (4) Primitive Formulas: The primitive formulas are identity and membership primitive formulas, of the form $t_{1}=t_{2}$ and $t_{1} \in t_{2}$, along with, for terms $t_{1}, \ldots, t_{n}$, formulas $\mathrm{P}\left(t_{1}, \ldots, t_{n}\right)$, constructed according to the sort restrictions. (5) Connectives \& $\mathcal{G}$ Quantifiers: We introduce the usual logical connectives $\neg, \wedge, \ldots$, and quantifiers $\forall$ and $\exists$ which can bind any variable. (6) Formulas: Formulas of $L\left(\sigma_{\in}\right)$ are primitive formulas, along with formulas obtained recursively by applying connectives and quantifiers, by the obvious modification of the usual definition of "formulas" for a 1-sorted first-order language. (7) Sublanguage $L(\sigma): L(\sigma)$ is the corresponding sublanguage over $\sigma$. (8) Sublanguage $L\left(\sigma\right.$ 「 $\left._{\text {atom }}\right): L(\sigma$ 「atom $)$ is the 1-sorted language over subsignature $\sigma \uparrow_{\text {atom }}$, in which all variables are atom variables, $a_{i}$.

\section{A.3 Quasi-logical Axioms}

Definition 22 (Boolean axioms). The following are called boolean axioms:

$$
\top \neq \perp \quad \forall \mathbf{b}(\mathbf{b}=\top \vee \mathbf{b}=\perp) .
$$

Definition 23 (Sort axioms). The following five formulas are called the sort axioms:

$$
\begin{gathered}
\text { SA1 }: \operatorname{atom}(x) \rightarrow \neg \operatorname{set}(x) . \\
\text { SA2 : atom }(x) \rightarrow \neg \operatorname{bool}(x) . \\
\text { SA3 }: \operatorname{bool}(x) \rightarrow \neg \operatorname{set}(x) . \\
\text { SA4 : bool }(x) \vee \operatorname{atom}(x) \vee \operatorname{set}(x) . \\
\text { SA5 }: x_{1} \in x_{2} \rightarrow \operatorname{set}\left(x_{2}\right) .
\end{gathered}
$$

Definition 24 (Subsort Axioms). The following schemes are called "Subsort Axioms":

$$
\begin{aligned}
\forall x \phi(x) & \rightarrow \forall a \phi(a) . \\
\forall x \phi(x) & \rightarrow \forall \mathbf{b} \phi(\mathbf{b}) . \\
\forall x \phi(x) & \rightarrow \forall X \phi(X) .
\end{aligned}
$$

where it is required that $\phi(a), \phi(\mathbf{b})$ and $\phi(X)$ be well-formed.

\section{References}

Andréka, H., Madarász, J. X., \& Németi, I., 2006: "Logical axiomatizations of spacetime. Samples from the literature". In A Prékopa \& E Molnár, eds., Non-Euclidean Geometries: János Bolyai Memorial Volume (Volume 581 of Mathematics and Its Applications), pages 155-185. Berlin: Springer Verlag.

\footnotetext{
$\overline{67}$ Usually what I shall call primitive terms and formulas are called atomic terms and atomic formulas. However, "atomic" has a different meaning in this paper.
} 
Andréka, H., Madarász, J. X., Németi, I., \& Székely, G., 2012: "A Logic Road from Special Relativity to General Relativity". Synthese 186(3) (Logic Meets Physics): 633-649.

Baker, A., 2005: "Are There Genuine Mathematical Explanations of Physical Phenomena?" Mind 114: 223-38.

Balzer, W., Moulines, C. U., \& Sneed, J. D., 1987: An Architectonic for Science: the Structuralist Approach. Dordrecht: Reidel.

Barwise, J., 1975: Admissible Sets and Structures: An Approach to Definability Theory (Perspectives in Mathematical Logic, Volume 7). Berlin: Springer-Verlag.

Barwise, J. \& Moss, L., 1996: Vicious Circles: On the Mathematics of Non-Wellfounded Phenomena (CSLI Lecture Notes: Number 60). Stanford: CSLI.

Batterman, R., 2002: The Devil in the Details: Asymptotic Reasoning in Explanation, Reduction, and Emergence. New York: Oxford University Press.

Bays, T., 2005: "Review: Set Theory and its Philosophy: A Critical Introduction (Michael Potter)". Notre Dame Philosophical Reviews https://ndpr.nd.edu/news/ set-theory-and-its-philosophy-a-critical-introduction/.

Benzmüller, C. \& Andrews, P., 2019: "Church's Type Theory". The Stanford Encyclopedia of Philosophy https://plato.stanford.edu/entries/type-theory-church/.

Bueno, O. \& Colyvan, M., 2011: "An Inferential Conception of the Application of Mathematics". Noûs 45 (2): 345-374.

Bueno, O. \& French, S., 2018: Applying Mathematics: Immersion, Inference, Interpretation. Oxford: Oxford University Press.

Burgess, J., 1984: "Synthetic Mechanics". Journal of Philosophical Logic 13: 319-95.

Burgess, J. \& Rosen, G., 1997: A Subject with No Object. Oxford: Clarendon Press.

Carnap, R., 1922: Der Raum: Ein Beitrag zur Wissenschaftslehre. Berlin: Reuther \& Reichard. English translation Carnap 2019, Rudolf Carnap: Early Writings edited by A.W. Carus, Michael Friedman, Wolfgang Kienzler, Alan Richardson, and Sven Schlotter (eds.) in The Collected Works of Rudolf Carnap, 1, New York: Oxford University Press.: 21-208.

Carnap, R., 1928: Der Logische Aufbau der Welt. Leipzig: Felix Meiner Verlag. English translation by Rolf A. George, 1967. The Logical Structure of the World. Pseudoproblems in Philosophy. University of California Press.

Carnap, R., 1956: "The Methodological Character of Theoretical Concepts". In H Feigl \& M Scriven, eds., Minnesota Studies in the Philosophy of Science I, pages 38-76. Minneapolis: University of Minnesota Press.

Carnap, R., 1958: "Beobachtungssprache und theoretische Sprache". Dialectica 12(34): 236-248. Translation: "Observational Language and Theoretical Language" in J. Hintikka (ed.) 1975, Rudolf Carnap. Logical Empiricist, Dordrecht: Reidel (pp. 75-85).

Carnap, R., 1966: The Philosophical Foundations of Physics. New York: Basic Books. 
Edited by Martin Gardner. Reprinted as An Introduction to the Philosophy of Science, New York: Dover Books, 1995.

Chihara, C., 1990: Constructibility and Mathematical Existence. Oxford: Oxford University Press.

Church, A., 1940: "A Formulation of the Simple Theory of Types". Journal of Symbolic Logic 5: 56-68.

Cieśliński, C., 2017: The Epistemic Lighteness of Truth: Deflationism and its Logic. Cambridge: Cambridge University Press.

Colyvan, M., 2001: The Indispensability of Mathematics. Oxford: Oxford University Press.

Colyvan, M., 2019: "Indispensability Arguments in the Philosophy of Mathematics". Stanford Encyclopedia of Mathematics First published Mon Dec 21, 1998; substantive revision Thu Feb 28, 2019.

https://plato.stanford.edu/entries/mathphil-indis/.

Coquand, T., 2018: "Type Theory". Stanford Encyclopedia of Philosophy

https://plato.stanford.edu/entries/type-theory/.

da Costa, N. \& Chuaqui, R., 1988: "On Suppes' Set-Theoretical Predicates". Erkenntnis 29: 95-112.

Einstein, A., 1921: "Geometry and Experience". Address to The Prussian Academy of Sciences (27 January 1921) Translated by G.B. Jeffrey \& W. Perret in ?.

Enderton, H., 2001: A Mathematical Introduction to Logic. Revised second edition. Harcourt/Academic Press.

Euler, L., 1736: "Solutio problematis ad geometriam situs pertinentis". Comment Acad Sci U Petrop 8: 128-140.

Feynman, R., 1965: The Character of the Physical Law. London: Penguin. New edition: 1992.

Field, H., 1980: Science Without Numbers. Princeton: Princeton University Press. Second edition, Oxford University Press (2016).

Forster, T., 1995: Set Theory with a Universal Set. Oxford: Oxford University Press. Oxford Logic Guides 31. Second edition.

Frege, G., 1884: Die Grundlagen der Arithmetic: Eine logisch-mathematische Untersuchung über den Begriff der Zahl. Breslau: Verlage Wilhelm Koebner. Translation by J.L. Austin as The Foundations of Arithmetic. Oxford: Blackwell, 1950.

French, S., 2020: There Are No Such Things As Theories. Oxford: Oxford University Press.

Friedman, M., 1982: "Review: The Scientific Image (by Bas C. Van Fraassen)". The Journal of Philosophy 79 (5): 274-283.

Frigg, R., 2012: "Models in Science". Stanford Encyclopedia of Philosophy First published Mon Feb 27, 2006; substantive revision Mon Jun 25, 2012.

https://plato.stanford.edu/entries/models-science/. 
Halbach, V., 2014: Axiomatic Theories of Truth. Cambridge: Cambridge University Press. First edition 2011. Revised edition 2014.

Halvorson, H., 2012: "What Scientific Theories Could Not Be". Philosophy of Science 79(2): $183-206$.

Halvorson, H., 2013: "The Semantic View, If Plausible, Is Syntactic". Philosophy of Science 80(3): 475-478.

Halvorson, H., 2016: "Scientific Theories". In P Humphreys, ed., Oxford Handbook of the Philosophy of Science. Oxford: Oxford University Press.

Harary, F., 1969: Graph Theory. Reading: Ma.: Addison-Wesley.

Hempel, C., ed., 1952: Fundamentals of Concept Formation in Empirical Science. Chicago: University of Chicago Press.

Hilbert, D., 1899: Grundlagen der Geometrie. Leibzig: Verlag Von B.G. Teubner. Translated (by E.J.Townsend) as The Foundations of Geometry. Chicago: Open Court.

Hölder, O., 1901: "Die Axiome der Quantität und die Lehre vom Mass"”. Berichten der mathematisch-physischen Classe der Königlisch Sächsischen Gesellschaft der Wissenschaften zu Leipzig, Mathematisch-Physikaliche Classe 53: 1-63. Translations of this work by Joel Michell and Catherine Ernst appear in ?, ?.

Holmes, M., 1998: Elementary Set Theory with a Universal Set. Louvain-la-Neuve: Université Catholique de Louvain, Département de Philosophie: Cahiers du Centre de Logique Vol 10.

Jech, T., 2002: Set Theory: The Third Millennium Edition. New York: Springer. 1st edition published in 1978 and the second edition in 1997. This is the 3rd revised and enlarged edition.

Jensen, R., 1968: "On the Consistency of a Slight (?) Modification of Quine's 'New Foundations"'. Synthese 19: 250-263.

Kaluza, T., 1921: "Zum Unitätsproblem in der Physik". Sitzungsber Preuss Akad Wiss Berlin (Math Phys) pages 966-972.

Kaye, R., 1991: Models of Peano Arithmetic. Oxford: Oxford University Press. Oxford Logic Guides 15.

Ketland, J., 1998: The Mathematicization of Nature. Ph.D Thesis: London School of Economics.

Ketland, J., 2011: "Nominalistic Adequacy". Proceedings of the Aristotelian Society 111: 201-217.

Klein, O., 1926: "Quantentheorie und fünfdimensionale Relativitätstheorie". Zeitschrift für Physik A 27 (12): 895-906.

Korbmacher, J. \& Schiemer, G., 2018: "What Are Structural Properties?" Philosophia Mathematica 26 (3): 295-323.

Krantz, D., Luce, R., Suppes, P., \& Tversky, A., 1971: Foundations of Measurement: Volume 1. Academic Press. 
Leitgeb, H. \& Carus, A., 2020: "Rudolf Carnap". Stanford Encyclopedia of Philosophy First published Mon Feb 24, 2020.

https://plato.stanford.edu/entries/carnap/.

Leng, M., 2010: Mathematics and Reality. Oxford: Oxford University Press.

Lewis, D., 1991: Parts of Classes. Oxford: Oxford University Press.

Lutz, S., 2012: "On a Straw Man in the Philosophy of Science - A Defense of the Received View". Hopos: The Journal of the International Society for the History of Philosophy of Science 2 (1): 77-120.

Lutz, S., 2014: "What's Right with a Syntactic Approach to Theories and Models?" Erkenntnis 79(8): 1475-1492.

Lutz, S., 2017: "What was the Syntax-Semantics Debate in the Philosophy of Science Sbout?" Philosophy and Phenomenological Research 95(2): 319-352.

Lyndon, R., 1959: "Properties preserved under homomorphism". Pacific Journal of Mathematics 9(1): 143-154.

Mancosu, P., 2018: "Explanation in Mathematics". Stanford Encyclopedia of Philosophy https://plato.stanford.edu/entries/mathematics-explanation/.

Manzano, M., 1996: Extensions of First-Order Logic. Cambridge: Cambridge University Press. Cambridge Tracts in Theoretical Computer Science.

McKinsey, J., Sugar, A., \& Suppes, P., 1953: "Axiomatic Foundation of Classical Particle Mechanics". Journal of Rational Mechanics and Analysis 2: 253-272.

Melia, J., 2000: "Weaseling Away The Indispensability Argument". Mind 109: 455-480.

Mendelson, E., 2010: An Introduction to Mathematical Logic. Boca Raton, FL.: Chapman \& Hall/CRC (Taylor \& Francis Group). Fifth edition (first edition published in 1964).

Menzel, C., 2014: "Wide Sets, ZFCU, and the Iterative Conception". Journal of Philosophy 111 (2): 57-83.

Muller, F., 2011: "Reflections on the Revolution at Stanford". Synthese 183(1): 87-114. Special issue: The Classical Model of Science II: The Axiomatic Method, the Order of Concepts and the Hierarchy of Sciences, edited by Arianne Betti, Willem de Jong and Marije Martijn.

Mycielski, J., 2013: "On the Formalization of Theories". Journal of Automated Reasoning 50: 211-216.

Narens, L., 1985: Abstract Measurement Theory. Cambridge, MA: MIT Press.

Pincock, C., 2007: "A Role for Mathematics in The Physical Sciences". Noûs 41(2): 253-275.

Pincock, C., 2012: Mathematics and Scientific Representation. Oxford: Oxford University Press.

Potter, M., 2004: Set Theory and its Philosophy: A Critical Introduction. Oxford: Oxford University Press. 
Przełęcki, M., 1969: The Logic of Empirical Theories. London: Routledge \& Kegan Paul. Putnam, H., 1967: "The Thesis That Mathematics is Logic". In R Schoenman, ed., Bertrand Russell, Philosopher of the Century. London: Allen and Unwin. Page references to reprint in ?.

Putnam, H., 1971: Philosophy of Logic. New York: Harper and Row. Reprinted in ?.

Quine, W., 1956: "Unification of Universes in Set Theory". The Journal of Symbolic Logic 21(3): 267-279.

Quine, W., 1986a: Philosophy of Logic. Cambridge, Ma.: Harvard University Press. Second, revised edition of ?.

Quine, W., 1986b: "Reply to Charles Parsons". In E Hahn \& P Schilpp, eds., The Philosophy of W.V. Quine (Volume 18: Library of Living Philosophers). Chicago: Open Court.

Ramsey, F., 1929: "Theories". In R Braithwaite, ed., Foundations of Mathematics. Amsterdam: Routledge and Kegan Paul (1931).

Reichenbach, H., 1924: Axiomatik der relativistischen Raum-Zeit-Lehre. Braunschweig: Friedr. Vieweg \& Sohn. Engl. transl. The Axiomatization of the Theory of Relativity, by M. Reichenbach with an introduction by W.C. Salmon. Berkeley-Los Angeles: University of California Press.

Resnik, M., 1997: Mathematics as a Science of Patterns. Oxford: Oxford University Press.

Roberts, F., 1985: Measurement Theory. Cambridge: Cambridge University Press.

Russell, B., 1903: The Principles of Mathematics. Cambridge: Cambridge University Press. Paperback edition: Routledge, London, 1992.

Russell, B., 1908: "Mathematical Logic as Based on the Theory of Types". American Journal of Mathematics 30 (3): 222-262.

Russell, B. \& Whitehead, A., 1912: Principia Mathematica: Vols I-III. Cambridge: Cambridge University Press.

Schlick, M., 1918: Allgemeine Erkenntnislehre. Berlin: Springer. 2nd edition 1925. Translated with introduction by Blumberg, A \& Feigl, H., as General Theory of Knowledge: Library of Exact Philosophy (Open Court, 1985).

Schmidt, F., 1938: "Über deductive Theorien mit mehreren Sorten von Grunddingen". Mathematische Annalen 115: 485-506.

Shapiro, S., 1997: Philosophy of Mathematics: Structure and Ontology. Oxford: Oxford University Press.

Simpson, S., 2009: Subsystems of Second-Order Arithmetic. Cambridge: Cambridge University Press. First edition, Berlin: Springer (1998).

Sneed, J., 1971: The Logical Structure of Mathematical Physics. Dordrecht: Reidel.

Stegmüller, W., 1976: The Structure and Dynamics of Theories. Berlin: Springer-Verlag. Steiner, M., 1998: The Applicability of Mathematics as a Philosophical Problem. Cam- 
bridge, Ma.: Harvard University Press.

Suppe, F., ed., 1977: The Structure of Scientific Theories. Chicago: University of Chicago Press.

Suppes, P., 1957: Introduction to Logic. New York: Van Nostrand Reinhold.

Suppes, P., 1960: "A Comparison of the Meaning and Use of Models in Mathematics and the Empirical Sciences". Synthese 12: 287-301.

Suppes, P., 1992: "Axiomatic Methods in Science". In M Carvallo, ed., Nature, cognition and system II, pages 205-232. Dordrecht: Kluwer.

Suppes, P., 2002: Representation and Invariance of Scientific Structures (CSLI Lecture Notes: 130). CSLI Publications.

Tal, E., 2015: "Measurement in Science". Stanford Encyclopedia of Phlosophy First published Mon Jun 15, 2015.

https://plato.stanford.edu/entries/measurement-science/.

Tarski, A., 1959: "What is Elementary Geometry?" In L Henkin, P Suppes, \& A Tarski, eds., The Axiomatic Method. Amsterdam: North Holland. Reprinted in ?.

van Fraassen, B., 1980: The Scientific Image. Oxford: Clarendon Press.

Wang, H., 1952: "The Logic of Many-Sorted Theories". The Journal of Symbolic Logic 17(2): 105-116.

Wenzel, M. et al., 2020: "The Isabelle/Isar Reference Manual". The manual is available online.

https://isabelle.in.tum.de/dist/Isabelle2020/doc/isar-ref.pdf.

Worrall, J., 1984: "An Unreal Image (Review of van Fraassen (1980))". British Journal for the Philosophy of Science 35: 65-80.

Zermelo, E., 1908: "Untersuchungen über die Grundlagen der Mengenlehre I". Mathematische Annalen 65. English translation by S. Bauer-Mengelberg, "Investigations in the Foundations of Set Theory I", in van Heijenoort (ed.) 1967. 